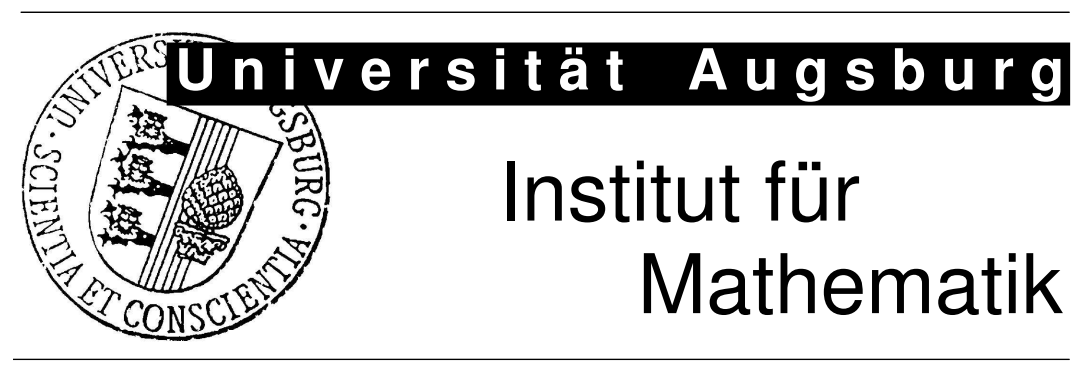

Stella David, Lothar Heinrich

Central Limit Theorem for the Integrated Squared Error of the Empirical Second-Order Product Density and Goodness-of-fit Tests for Stationary Point Processes 


\section{Impressum:}

\section{Herausgeber:}

Institut für Mathematik

Universität Augsburg

86135 Augsburg

http://www.math.uni-augsburg.de/pages/de/forschung/preprints.shtml

\section{ViSdP:}

Stella David

Institut für Mathematik

Universität Augsburg

86135 Augsburg

Preprint: Sämtliche Rechte verbleiben den Autoren (C) 2010 


\title{
Central limit theorem for the integrated squared error of the empirical second-order product density and goodness-of-fit tests for stationary point processes
}

\author{
Stella David and Lothar Heinrich
}

\begin{abstract}
Spatial point processes are mathematical models for irregular or random point patterns in the $d$-dimensional space, where usually $d=2$ or $d=3$ in applications. The second-order product density and its isotropic analogue, the pair correlation function, are important tools for analyzing stationary point processes. In the present work we derive central limit theorems for the integrated squared error (ISE) of the empirical second-order product density and for the ISE of the empirical pair correlation function for expanding observation windows. The proof techniques are based on higher-order cumulant measures and the Brillinger-mixing property of the underlying point processes. The obtained Gaussian limits are used for constructing asymptotic goodness-of-fit tests for checking point process hypotheses even in the non-Poissonian case.

AMS 2000 Mathematics Subject Classification: Primary: 60G55, 62M30, 60F05; Secondary: 62G10, 62G20

Keywords: Second-order analysis of point processes, pair correlation function, Brillingermixing, cumulant measures, asymptotic variance, model specification test
\end{abstract}

\section{Introduction}

An important aim of point process statistics is to find a mathematical model that gives a satisfactory description of an observed point pattern. With such mathematical models one can, for instance, draw conclusions about properties of certain materials or tissues. For stationary point processes mainly second-order statistics such as Ripley's $K$-function and the pair correlation function are used for verifying or rejecting hypothetical point process models by visual inspection or simulation tests, see e.g. Baddeley et al. [1], Cressie [3], Diggle [6], Illian et al. [13], and Stoyan et al. [19]. Often these investigations focus on complete spatial randomness, see e.g. Grabarnik and Chiu [7], Ho and Chiu [12], and Zimmerman [22]. Most tests used in applications are based on heuristic considerations rather than on mathematical models. This is mainly due to the latter models' complexity caused by dimension and by stochastic dependencies of neighboring areas. In the present work we will use the second-order product density and its isotropic analogue, the pair correlation function, to construct goodness-of-fit tests for a wide class of stationary point processes. Based on one realization of a point process in a convex observation window expanding in every direction we study the integrated squared error (ISE) of the estimated second-order product density and the ISE of the estimated pair correlation function. The asymptotic behavior of the ISE of probability density estimators has been studied e.g. by Hall [9] who derived central limit 
theorems (CLTs) for the ISE for independent random variables and by Takahata and Yoshihara [21] who extended Hall's result to absolutely regular random sequences. We will derive CLTs for the ISE of the empirical second-order product density and for the ISE of the empirical pair correlation function in the setting of Brillinger-mixing point processes. The limiting normal distribution will solely depend on the underlying hypothetical second-order quantity, the intensity of the point process, and the kernel function. This allows the construction of distribution-free testing procedures.

Firstly, we will define some basic notions. Let $[N, \mathcal{N}]$ denote the measurable space of all locally finite counting measures on the $d$-dimensional Euclidean space $\mathbb{R}^{d}$ equipped with its $\sigma$-algebra $\mathfrak{B}\left(\mathbb{R}^{d}\right)$ of Borel sets. A point process (shortly PP) on $\mathbb{R}^{d}$ is defined as a measurable mapping $\Psi$ from a probability space $[\Omega, \mathcal{A}, \mathbb{P}]$ into $[N, \mathcal{N}]$. Throughout in this paper we assume that $\Psi$ is simple, i.e. $\mathbb{P}\left(\Psi(\{x\}) \leq 1\right.$ for all $x \in \mathbb{R}^{d}$, and stationary, i.e. $\Psi(B+x)$ and $\Psi(B)$ have the same distribution for any $x \in \mathbb{R}^{d}$ and $B \in \mathfrak{B}\left(\mathbb{R}^{d}\right)$ (for a test on stationarity see Guan [8]). Let $\mathbb{E}$ and Var denote expectation and variance, respectively, with respect to $\mathbb{P}$. Let $P=\mathbb{P} \circ \Psi^{-1}$ denote the probability measure on $[N, \mathcal{N}]$ induced by $\Psi$ and we will briefly write $\Psi \sim P$. If $\mathbb{E} \Psi^{k}(B)<\infty$ for all bounded Borel sets $B$, then there exist the $k$ th-order factorial moment measure $\alpha^{(k)}$ and the $k$ th-order factorial cumulant measure $\gamma^{(k)}$ on $\left[\left(\mathbb{R}^{d}\right)^{k}, \mathfrak{B}\left(\left(\mathbb{R}^{d}\right)^{k}\right)\right]$ defined by

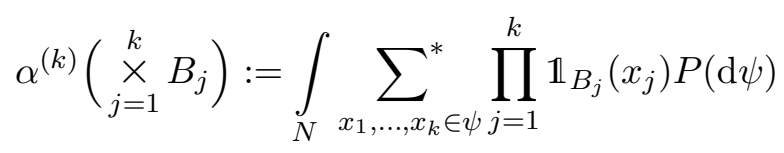

and

$$
\gamma^{(k)}\left(\underset{j=1}{\stackrel{k}{\times}} B_{j}\right):=\sum_{\ell=1}^{k}(-1)^{\ell-1}(\ell-1) ! \sum_{\substack{K_{1} \cup \ldots \cup K_{\ell} \\=\{1, \ldots, k\}}} \prod_{j=1}^{\ell} \alpha^{\left(\# K_{j}\right)}\left(\underset{\substack{\times \\ k_{j} \in K_{j}}}{\times} B_{k_{j}}\right)
$$

with $B_{1}, \ldots, B_{k} \in \mathfrak{B}\left(\mathbb{R}^{d}\right)$, respectively. Here the abbreviation " $x \in \psi$ " means " $x \in \mathbb{R}^{d}: \psi(\{x\})>$ 0 ". Further, $\sum^{*}$ denotes summation over summands with index tuples having pairwise distinct components. The sum $\sum_{K_{1} \cup \ldots \cup K_{\ell}}$ is taken over all partitions of the set $\{1,2, \ldots, k\}$ into $\ell$ disjoint non-empty subsets $K_{j}$ and $\# K_{j}$ denotes the cardinality of $K_{j}$. If $\Psi \sim P$ is stationary with intensity $\lambda>0$ the $k$ th-order reduced factorial moment measure $\alpha_{\text {red }}^{(k)}$ is implicitly defined by the disintegration

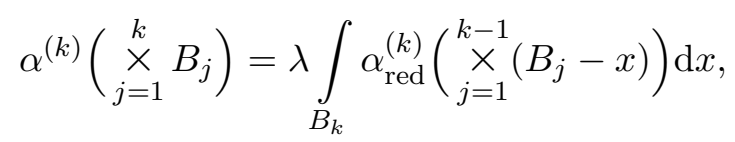

see Daley and Vere-Jones [4, p. 238]. Analogously, the disintegration

$$
\gamma^{(k)}\left(\underset{j=1}{\stackrel{k}{\times}} B_{j}\right)=\lambda \int_{B_{k}} \gamma_{\text {red }}^{(k)}\left(\underset{j=1}{\stackrel{k-1}{\times}}\left(B_{j}-x\right)\right) \mathrm{d} x
$$

defines the kth-order reduced factorial cumulant measure $\gamma_{\text {red }}^{(k)}$.

The total variation measure $\left|\gamma_{\text {red }}^{(k)}\right|$ is defined by $\left|\gamma_{\text {red }}^{(k)}\right|()=.\left(\gamma_{\text {red }}^{(k)}\right)^{+}()+.\left(\gamma_{\text {red }}^{(k)}\right)^{-}($.$) , where the$ measures $\left(\gamma_{\text {red }}^{(k)}\right)^{+}$and $\left(\gamma_{\text {red }}^{(k)}\right)^{-}$are given by the Jordan decomposition $\gamma_{\text {red }}^{(k)}()=.\left(\gamma_{\text {red }}^{(k)}\right)^{+}()-$. $\left(\gamma_{\text {red }}^{(k)}\right)^{-}($.$) . The total variation of \gamma_{\text {red }}^{(k)}$ is defined by $\left.\left\|\gamma_{\text {red }}^{(k)}\right\|:=\left|\gamma_{\text {red }}^{(k)}\right|\left(\left(\mathbb{R}^{d}\right)^{k-1}\right)\right)$. 
A stationary $\mathrm{PP} \Psi \sim P$ in $\mathbb{R}^{d}$ satisfying $\mathbb{E} \Psi^{k}\left([0,1]^{d}\right)<\infty$ for some $k \geq 2$ is said to be $\mathrm{B}_{k}$-mixing if

$$
\left\|\gamma_{\text {red }}^{(j)}\right\|=\int_{\left(\mathbb{R}^{d}\right)^{j-1}}\left|\gamma_{\text {red }}^{(j)}\left(\mathrm{d}\left(x_{1}, \ldots, x_{j-1}\right)\right)\right|<\infty \quad \text { for } j=2, \ldots, k .
$$

If $\Psi$ is $\mathrm{B}_{k}$-mixing for all $k \geq 2, \Psi$ is called Brillinger-mixing or $\mathrm{B}_{\infty}$-mixing, see Brillinger [2] (for $d=1$ ) or Ivanoff [14]. Heinrich [10] and Heinrich and Schmidt [11] state conditions on several classes of PPes for being $\mathrm{B}_{\infty}$-mixing.

If the $k$ th-order reduced factorial moment measure $\alpha_{\text {red }}^{(k)}$ is absolutely continuous with respect to the Lebesgue measure on $\left[\left(\mathbb{R}^{d}\right)^{k-1}, \mathfrak{B}\left(\left(\mathbb{R}^{d}\right)^{k-1}\right)\right]$, then its Lebesgue density $\varrho^{(k)}$ is given by

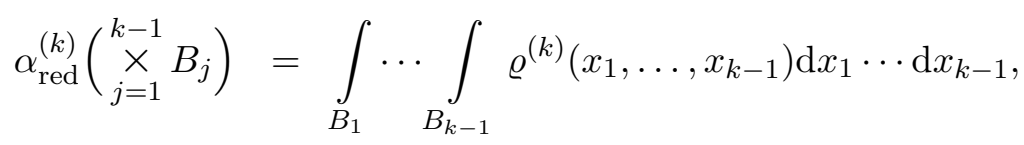

where $B_{1}, \ldots, B_{k-1} \in \mathfrak{B}\left(\mathbb{R}^{d}\right)$, and is called the kth-order reduced product density, henceforth abbreviated as $k$ th-order product density.

If the $k$ th-order reduced factorial cumulant measure $\gamma_{\text {red }}^{(k)}$ is absolutely continuous with respect to the Lebesgue measure on $\left[\left(\mathbb{R}^{d}\right)^{k-1}, \mathfrak{B}\left(\left(\mathbb{R}^{d}\right)^{k-1}\right)\right]$, then its Lebesgue density $c^{(k)}$ is given by

$$
\gamma_{\text {red }}^{(k)}\left(\underset{j=1}{\stackrel{k-1}{\times} B_{j}}\right)=\int_{B_{1}} \ldots \int_{B_{k-1}} c^{(k)}\left(x_{1}, \ldots, x_{k-1}\right) \mathrm{d} x_{1} \cdots \mathrm{d} x_{k-1},
$$

where $B_{1}, \ldots, B_{k-1} \in \mathfrak{B}\left(\mathbb{R}^{d}\right)$, and is called the $k$ th-order reduced cumulant density, henceforth abbreviated as $k$ th-order cumulant density.

In this paper we will focus on the second-order product density $\varrho^{(2)}$, henceforth abbreviated as product density $\varrho$, and its isotropic analogue, the pair correlation function (PCF), defined by

$$
g(r):=\frac{\varrho(x)}{\lambda},
$$

where $r=\|x\|, x \in \mathbb{R}^{d}$, and $\lambda$ is the intensity of the stationary PP.

The rest of the paper is organized as follows. Section 2 introduces the estimators for the product density and the PCF and their ISEs. In Section 3 we derive CLTs for these ISEs. In Section 4, these results are used for constructing asymptotic goodness-of-fit tests. The proofs of the results in Section 4 are carried out in Section 5.

\section{Integrated squared error of the empirical product density and of the empirical pair correlation function}

In this section we will present the estimators for the product density and the PCF and their ISEs and formulate some conditions needed for our asymptotic results in the next sections.

Let $\rho(W):=\sup \left\{r \geq 0: b(x, r) \subset W, x \in \mathbb{R}^{d}\right\}$ denote the inradius of the set $W \subset \mathbb{R}^{d}$, where $b(x, r):=\left\{y \in \mathbb{R}^{d}:\|y-x\| \leq r\right\}$ is the ball with radius $r \geq 0$ centered at $x \in \mathbb{R}^{d}$. Let $|$.$| denote$ the Lebesgue measure on $\left[\mathbb{R}^{d}, \mathfrak{B}\left(\mathbb{R}^{d}\right)\right]$ and let $\omega_{d}=|b(o, 1)|$. The following condition is needed for $s=1$ or $s=d$. 
Condition $(\mathcal{C}(s))$. (i) The sequence of observation windows $\left(W_{n}\right)_{n \in \mathbb{N}}$ is an increasing sequence of convex and compact sets in $\mathbb{R}^{d}$ with $\rho\left(W_{n}\right) \underset{n \rightarrow \infty}{\longrightarrow} \infty$,

(ii) the sequence of bandwidths $\left(b_{n}\right)_{n \in \mathbb{N}}$ is a decreasing sequence of positive real numbers satisfying $b_{n} \underset{n \rightarrow \infty}{\longrightarrow} 0$ and $b_{n}^{s}\left|W_{n}\right| \underset{n \rightarrow \infty}{\longrightarrow} \infty$, and

(iii) the kernel function $k: \mathbb{R}^{s} \rightarrow \mathbb{R}$ is bounded with bounded support, symmetric (i.e., $k(x)=$ $k(-x)$ for every $\left.x \in \mathbb{R}^{s}\right)$, and satisfies $\int_{\mathbb{R}^{s}} k(x) \mathrm{d} x=1$.

The following definition of a kernel-type estimator for the product density goes back to Krickeberg [17]. The speed of convergence of this estimator has been studied in Jolivet [16].

Definition 2.1. Let $\left(W_{n}\right)_{n \in \mathbb{N}},\left(b_{n}\right)_{n \in \mathbb{N}}$ and $k$ satisfy Condition $\mathcal{C}(d)$. Let the $P P \Psi \sim P$ in $\mathbb{R}^{d}$ be stationary and assume its product density $\varrho$ to exist. Then we define

$$
\hat{\varrho}_{n}(t):=\frac{1}{b_{n}^{d}\left|W_{n}\right|} \sum_{x_{1}, x_{2} \in \Psi}^{*} \mathbb{1}_{W_{n}}\left(x_{1}\right) k\left(\frac{x_{2}-x_{1}-t}{b_{n}}\right)
$$

as an estimator for $\lambda \varrho(t)$ for $t \in \mathbb{R}^{d}$.

Definition 2.2. Let $\left(W_{n}\right)_{n \in \mathbb{N}},\left(b_{n}\right)_{n \in \mathbb{N}}$ and $k$ satisfy Condition $\mathcal{C}(1)$. Let the $P P \Psi \sim P$ in $\mathbb{R}^{d}$ be stationary and assume its PCF $g$ to exist. Then we define

$$
\hat{g}_{n}(r)=\frac{1}{b_{n}\left|W_{n}\right| d \omega_{d}} \sum_{x_{1}, x_{2} \in \Psi}^{*} \frac{\mathbb{1}_{W_{n}}\left(x_{1}\right)}{\left\|x_{2}-x_{1}\right\|^{d-1}} k\left(\frac{\left\|x_{2}-x_{1}\right\|-r}{b_{n}}\right)
$$

as an estimator for $\lambda^{2} g(r)$ for $r \in[0, \infty)$.

For a discussion of estimators for the PCF with regard to bias and variance see Stoyan and Stoyan [20].

The integrated squared error (ISE) of the product density estimator is defined by

$$
I_{n}(K):=\int_{K}\left(\hat{\varrho}_{n}(t)-\lambda \varrho(t)\right)^{2} \mathrm{~d} t,
$$

where $K \in \mathfrak{B}\left(\mathbb{R}^{d}\right),|K|>0$, is a bounded set. Likewise, the ISE of the PCF estimator is defined by

$$
J_{n}(K):=\int_{K}\left(\hat{g}_{n}(r)-\lambda^{2} g(r)\right)^{2} \mathrm{~d} r,
$$

where $K \in \mathfrak{B}((0, \infty)),|K|>0$, is a bounded set.

Condition $\left(\mathcal{C}_{\varrho}(K)\right)$. Let $\Psi \sim P$ be a stationary $P P$ in $\mathbb{R}^{d}$ with product density $\varrho$ such that, for some $K \in \mathfrak{B}\left(\mathbb{R}^{d}\right)$ and some $\varepsilon>0$,

(i) the first-order partial derivatives of the product density @ are uniformly Lipschitz-continuous in $K \oplus b(o, \varepsilon)$ and 
(ii) the third- and fourth-order cumulant densities $c^{(3)}$ and $c^{(4)}$ exist and satisfy

$$
\sup _{u, v \in K \oplus b(o, \varepsilon)}\left|c^{(3)}(u, v)\right|<\infty \quad \text { and } \quad \sup _{u, v \in K \oplus b(o, \varepsilon)} \int_{\mathbb{R}^{d}}\left|c^{(4)}(u, w, v+w)\right| \mathrm{d} w<\infty .
$$

Condition $\left(\mathcal{C}_{g}(K)\right)$. Let $\Psi \sim P$ be a stationary $P P$ in $\mathbb{R}^{d}$ with $P C F g$ such that, for some $K \in \mathfrak{B}((0, \infty))$ and some $\varepsilon>0$,

(i) the first derivative of the PCF $g$ is uniformly Lipschitz-continuous in $K \oplus(-\varepsilon, \varepsilon)$ and

(ii) the third- and fourth-order cumulant densities $c^{(3)}$ and $c^{(4)}$ exist and satisfy

$$
\sup _{\substack{u, v \in \mathbb{R}^{d} \\\|u\|,\|v\| \in K \oplus(-\varepsilon, \varepsilon)}}\left|c^{(3)}(u, v)\right|<\infty \quad \text { and } \quad \sup _{\substack{u, v \in \mathbb{R}^{d}: \\\|u\|,\|v\| \in K \oplus(-\varepsilon, \varepsilon) \mathbb{R}^{d}}} \int\left|c^{(4)}(u, w, v+w)\right| \mathrm{d} w<\infty .
$$

\section{Central limit theorems}

In this section we will present asymptotic representations of the mean and the variance of the ISE $I_{n}(K)$ of the product density estimator. Then we will state a CLT for $I_{n}(K)$ for $\mathrm{B}_{\infty}$-mixing PPes. The proofs can be found in Section 5. The results for the ISE $J_{n}(K)$ of the PCF estimator can be shown analogously and will be given without proof.

The following lemma gives an asymptotic representation of the mean of the ISE $I_{n}(K)$ of the product density estimator.

Lemma 3.1. Let $\Psi \sim P$ be a $\mathrm{B}_{4}$-mixing $P P$ in $\mathbb{R}^{d}$ with intensity $\lambda$ and product density $\varrho$ satisfying Condition $\mathcal{C}_{\varrho}(K)$. Let $\left(W_{n}\right)_{n \in \mathbb{N}},\left(b_{n}\right)_{n \in \mathbb{N}}$ and $k$ satisfy Condition $\mathcal{C}(d)$. Then we have

$$
b_{n}^{d}\left|W_{n}\right| \mathbb{E} \int_{K}\left(\varrho_{n}(t)-\lambda \varrho(t)\right)^{2} \mathrm{~d} t=\lambda \int_{K} \varrho(t) \mathrm{d} t \int_{\mathbb{R}^{d}} k^{2}(x) \mathrm{d} x+\mathcal{O}\left(b_{n}^{d \wedge 2}\right)+\mathcal{O}\left(b_{n}^{d+4}\left|W_{n}\right|\right)
$$

as $n \rightarrow \infty$ for all bounded $K \in \mathfrak{B}\left(\mathbb{R}^{d}\right),|K|>0$.

To express the asymptotic variance of the ISE $I_{n}(K)$ in the next lemma we need the convolution $k * k$ of the kernel function $k$ with itself.

Lemma 3.2. Let $\Psi \sim P$ be a $\mathrm{B}_{8}$-mixing $P P$ in $\mathbb{R}^{d}$ with intensity $\lambda$ and product density $\varrho$ satisfying Condition $\mathcal{C}_{\varrho}(K)$. Let $\left(W_{n}\right)_{n \in \mathbb{N}},\left(b_{n}\right)_{n \in \mathbb{N}}$ and $k$ satisfy Condition $\mathcal{C}(d)$. Furthermore let $b_{n}^{d+4}\left|W_{n}\right| \underset{n \rightarrow \infty}{\longrightarrow} 0$. Then we have

$$
\operatorname{Var}\left(b_{n}^{d / 2}\left|W_{n}\right| \int_{K}\left(\hat{\varrho}_{n}(t)-\lambda \varrho(t)\right)^{2} \mathrm{~d} t\right) \underset{n \rightarrow \infty}{\longrightarrow} \sigma^{2}
$$

with

$$
\sigma^{2}:=2 \lambda^{2}\left(\int_{K} \varrho^{2}(t) \mathrm{d} t+\int_{K \cap(-K)} \varrho^{2}(t) \mathrm{d} t\right) \int_{\mathbb{R}^{d}}(k * k)^{2}(t) \mathrm{d} t
$$

for all bounded $K \in \mathfrak{B}\left(\mathbb{R}^{d}\right),|K|>0$. 
Now we state a CLT for the ISE of the product density estimator in the setting of $\mathrm{B}_{\infty}$-mixing PPes. The result will be proved in Section 5 by showing the cumulants of order $k \geq 3$ of the suitably scaled ISE to converge to zero.

The notation $\underset{n \rightarrow \infty}{\stackrel{\mathrm{d}}{\longrightarrow}}$ stands for weak convergence and $\mathcal{N}\left(\mu, \sigma^{2}\right)$ for the univariate normal distribution with mean $\mu \in \mathbb{R}$ and variance $\sigma^{2}>0$.

Theorem 3.3. Let $\Psi \sim P$ be a $\mathrm{B}_{\infty}$-mixing $P P$ in $\mathbb{R}^{d}$ with intensity $\lambda$ and product density $\varrho$ satisfying Condition $\mathcal{C}_{\varrho}(K)$. Let all cumulant densities $c^{(k)}, k \geq 2$, exist. Let $\left(W_{n}\right)_{n \in \mathbb{N}},\left(b_{n}\right)_{n \in \mathbb{N}}$ and $k$ satisfy Condition $\mathcal{C}(d)$, and, in addition, $b_{n}^{d+4}\left|W_{n}\right| \underset{n \rightarrow \infty}{\longrightarrow} 0$. Then we have

$$
b_{n}^{d / 2}\left|W_{n}\right|\left(I_{n}(K)-\mathbb{E} I_{n}(K)\right) \underset{n \rightarrow \infty}{\stackrel{\mathrm{d}}{\longrightarrow}} \mathcal{N}\left(0, \sigma^{2}\right)
$$

for all bounded $K \in \mathfrak{B}\left(\mathbb{R}^{d}\right),|K|>0$, with $\sigma^{2}$ given in Lemma 3.2.

Now we present an asymptotic representation for the mean of the ISE $J_{n}(K)$ of the PCF estimator and a CLT for the centered and suitably scaled ISE $J_{n}(K)$.

Lemma 3.4. Let $\Psi \sim P$ be a $\mathrm{B}_{4}$-mixing $P P$ in $\mathbb{R}^{d}$ with intensity $\lambda$ and $P C F g$ satisfying Condition $\mathcal{C}_{g}(K)$. Let $\left(W_{n}\right)_{n \in \mathbb{N}},\left(b_{n}\right)_{n \in \mathbb{N}}$ and $k$ satisfy Condition $\mathcal{C}(1)$. Then we have

$$
b_{n}\left|W_{n}\right| \mathbb{E} \int_{K}\left(\hat{g}_{n}(r)-\lambda^{2} g(r)\right)^{2} \mathrm{~d} r=2 \lambda^{2} \int_{K} \frac{g(r)}{d \omega_{d} r^{d-1}} \mathrm{~d} r \int_{\mathbb{R}} k^{2}(x) \mathrm{d} x+\mathcal{O}\left(b_{n}\right)+\mathcal{O}\left(b_{n}^{5}\left|W_{n}\right|\right)
$$

as $n \rightarrow \infty$ for all bounded $K \in \mathfrak{B}((0, \infty)),|K|>0$.

Theorem 3.5. Let $\Psi \sim P$ be a $\mathrm{B}_{\infty}$-mixing $P P$ in $\mathbb{R}^{d}$ with intensity $\lambda$ and $P C F$ g satisfying Condition $\mathcal{C}_{g}(K)$. Let all cumulant densities $c^{(k)}, k \geq 2$, exist. Let $\left(W_{n}\right)_{n \in \mathbb{N}},\left(b_{n}\right)_{n \in \mathbb{N}}$ and $k$ satisfy Condition $\mathcal{C}(1)$, and, in addition, $b_{n}^{5}\left|W_{n}\right| \underset{n \rightarrow \infty}{\longrightarrow} 0$. Then we have

$$
b_{n}^{1 / 2}\left|W_{n}\right|\left(J_{n}(K)-\mathbb{E} J_{n}(K)\right) \underset{n \rightarrow \infty}{\stackrel{\mathrm{d}}{\longrightarrow}} \mathcal{N}\left(0, \tau^{2}\right)
$$

with

$$
\tau^{2}:=8 \lambda^{4} \int_{K}\left(\frac{g(r)}{d \omega_{d} r^{d-1}}\right)^{2} \mathrm{~d} r \int_{\mathbb{R}}(k * k)^{2}(x) \mathrm{d} x
$$

for all bounded $K \in \mathfrak{B}((0, \infty)),|K|>0$.

\section{Asymptotic goodness-of-fit tests}

Given a realization of a $\mathrm{PP} \Psi \sim P$ in a sufficiently large observation window, one is interested in whether a hypothetical distribution $P_{0}$ of a $\mathrm{PP}$ is a good fit for the unknown true distribution $P$ (see e.g. Diggle [5]). In this section we will use the CLTs in Section 3 for constructing asymptotic goodness-of-fit tests for PPes in order to get a decision rule for the non-parametric test problem $H_{0}: P=P_{0}$ versus $H_{1}: P \neq P_{0}$. The test statistic is based on the ISE which uses only the intensity and the product density (or the PCF) in the set $K$ as information from the PP $\Psi$. 
Although second-order quantities do not characterize the distribution of the PP they still give a rather informative description of the point pattern and are therefore an appropriate basis for goodness-of-fit tests.

Compared with existing goodness-of-fit tests, our tests have the advantage that they are theoretically motivated (not based on simulations) and, at the same time, can be applied to a wide class of PPes (not only Poisson processes).

Now we will sketch how Theorem 3.3 and Theorem 3.5 are used for constructing asymptotic goodness-of-fit tests. For $d \in\{1,2,3\}$ we can use Lemma 3.1 to get a simple representation for the mean of the ISE $I_{n}(K)$. The choice of a symmetric set $K$ will simplify the variance. For the simplification of the mean of the ISE $J_{n}(K)$ of the PCF estimator, see Lemma 3.4, there is no restriction to certain dimensions necessary. In the following, $z_{q}$ denotes the $q$-quantile of the standard normal distribution.

\section{Testing $H_{0}: P=P_{0}$ versus $H_{1}: P \neq P_{0}$ using $I_{n}(K)$}

Let $\Psi \sim P$ be a PP in $\mathbb{R}^{d}, d \in\{1,2,3\}$, and let the hypothetical PP $\Psi_{0} \sim P_{0}$ with intensity $\lambda_{0}$ and product density $\varrho_{0}$ satisfy the assumptions of Theorem 3.3. Furthermore let $b_{n}^{d / 2+4}\left|W_{n}\right| \underset{n \rightarrow \infty}{\longrightarrow} 0$ and let $K \in \mathfrak{B}\left(\mathbb{R}^{d}\right)$ be bounded and symmetric with $|K|>0$. Then

$$
T_{n}=\frac{b_{n}^{d / 2}\left|W_{n}\right|}{\sigma_{0}^{2}}\left(\int_{K}\left(\varrho_{n}(t)-\lambda_{0} \varrho_{0}(t)\right)^{2} \mathrm{~d} t-\frac{\lambda_{0}}{b_{n}^{d}\left|W_{n}\right|} \int_{K} \varrho_{0}(t) \mathrm{d} t \int_{\mathbb{R}} k^{2}(x) \mathrm{d} x\right)
$$

with

$$
\sigma_{0}^{2}=4 \lambda_{0}^{2} \int_{K} \varrho_{0}^{2}(t) \mathrm{d} t \int_{\mathbb{R}^{d}}(k * k)^{2}(t) \mathrm{d} t
$$

lies in $\left[-z_{1-\alpha / 2}, z_{1-\alpha / 2}\right]$ with probability $1-\alpha$ approximately. Given a significance level $\alpha \in(0,1)$ we reject the null hypothesis $H_{0}: P=P_{0}$ if $T_{n}$ lies outside $\left[-z_{1-\alpha / 2}, z_{1-\alpha / 2}\right]$.

\section{Testing $H_{0}: P=P_{0}$ versus $H_{1}: P \neq P_{0}$ using $J_{n}(K)$}

Let $\Psi \sim P$ be a PP in $\mathbb{R}^{d}$ and let the hypothetical PP $\Psi_{0} \sim P_{0}$ with intensity $\lambda_{0}$ and PCF $g_{0}$ satisfy the assumptions of Theorem 3.5. Furthermore let $b_{n}^{9 / 2}\left|W_{n}\right| \underset{n \rightarrow \infty}{\longrightarrow} 0$ and let $K \in \mathfrak{B}((0, \infty))$ be bounded with $|K|>0$. Then

$$
T_{n}=\frac{b_{n}^{1 / 2}\left|W_{n}\right|}{\tau_{0}^{2}}\left(\int_{K}\left(\hat{g}_{n}(r)-\lambda_{0}^{2} g_{0}(r)\right)^{2} \mathrm{~d} r-\frac{2 \lambda_{0}^{2}}{b_{n}\left|W_{n}\right|} \int_{K} \frac{g_{0}(r)}{d \omega_{d} r^{d-1}} \mathrm{~d} r \int_{\mathbb{R}} k^{2}(x) \mathrm{d} x\right)
$$

with

$$
\tau_{0}^{2}=8 \lambda_{0}^{4} \int_{K}\left(\frac{g_{0}(r)}{d \omega_{d} r^{d-1}}\right)^{2} \mathrm{~d} r \int_{\mathbb{R}}(k * k)^{2}(x) \mathrm{d} x
$$

lies in $\left[-z_{1-\alpha / 2}, z_{1-\alpha / 2}\right]$ with probability $1-\alpha$ approximately. Given a significance level $\alpha \in(0,1)$ we reject the null hypothesis $H_{0}: P=P_{0}$ if $T_{n}$ lies outside $\left[-z_{1-\alpha / 2}, z_{1-\alpha / 2}\right]$.

An important question concerning the applicability of our asymptotic goodness-of-fit tests is how large the observation window has to be for a satisfactory approximation in the CLT. An answer 
may be found through simulation studies. The approximation will depend on several factors such as the distribution of the underlying $\mathrm{PP}$ - in particular the intensity and the product density or $\mathrm{PCF}$ - , the choice of the bandwidth and the kernel function, and the choice of the set $K$. Given a hypothetical distribution $P_{0}$ and the associated test problem $H_{0}: P=P_{0}$ versus $H_{1}: P \neq P_{0}$ it is obvious how to investigate the type-I error (that is, the probability of rejecting the null hypothesis when it is actually true) by simulation studies. The type-II error (that is, the probability of not rejecting the null hypothesis when the alternative hypothesis is actually true) is difficult to handle since the true distribution $P$ can differ from $P_{0}$ in many different ways. Hence the type-II error can only be studied for some special cases. For example, if $P=\Pi_{\lambda}$ and $P_{0}=\Pi_{\lambda_{0}}$ with $\lambda \neq \lambda_{0}$, an investigation of the type-II error for different combinations of $\lambda$ and $\lambda_{0}$ is a sensitivity analysis of the test procedure with respect to the intensity of the underlying Poisson process. Another example of such a sensitivity analysis is given in Grabarnik and Chiu [7] who consider the null hypothesis of a Poisson process and the alternative hypothesis of a mixture of a conditional Strauss PP and Matérn's cluster process.

Note that the intensity $\lambda_{0}$ must be known -if $I_{n}(K)$ is replaced by $\int_{K}\left(\hat{\varrho}_{n}(t)-\widehat{\left(\lambda_{0}\right)_{n}} \varrho(t)\right)^{2} \mathrm{~d} t$, the limiting distribution may differ (as it is the case for the Kolmogorov-Smirnov statistic if the parameters are estimated). However, the intensity $\lambda_{0}$ occurring in the mean and the variance of $T_{n}$ can be replaced by a consistent estimator $\widehat{\left(\lambda_{0}\right)_{n}}$ due to Slutsky's theorem. Another problem might arise if the product density $\varrho_{0}$ of the hypothetical PP $\Psi_{0}$ is not known explicitly. Nevertheless, our tests can be applied if $\varrho_{0}$ is replaced by an estimator $\widehat{\left(\varrho_{0}\right)_{n}}$ achieved by simulation of the null hypothesis model. It should be ensured that $\left.\widehat{(\varrho}_{0}\right)_{n}$ meets Condition $\mathcal{C}_{\varrho}(K)(\mathrm{i})$, e.g., by using a kernel function that satisfies $\mathcal{C}_{\varrho}(K)(\mathrm{i})$. Analogous considerations apply to the test based on the PCF.

\section{Proofs}

The normal convergence of the centered and suitably scaled ISE $I_{n}(K)$ is proved by showing all cumulants of order three and higher to converge to zero. Lemmas 5.1 and 5.2 will lead to a representation for the cumulants of the ISE of the estimated product density. This representation can also be used for deriving the asymptotic variance of the ISE. We start with the proof for the asymptotic representation of the mean of the ISE $I_{n}(K)$.

Proof of Lemma 3.1. By Fubini's theorem we have

$$
\mathbb{E} \int_{K}\left(\varrho_{n}(t)-\lambda \varrho(t)\right)^{2} \mathrm{~d} t=\int_{K} \operatorname{Var}\left(\hat{\varrho}_{n}(t)\right) \mathrm{d} t+\int_{K}\left(\mathbb{E} \hat{\varrho}_{n}(t)-\lambda \varrho(t)\right)^{2} \mathrm{~d} t .
$$

For the second summand we get

$$
b_{n}^{d}\left|W_{n}\right| \int_{K}\left(\mathbb{E} \hat{\varrho}_{n}(t)-\lambda \varrho(t)\right)^{2} \mathrm{~d} t=b_{n}^{d}\left|W_{n}\right| \lambda^{2} \int_{K}\left(\int_{\mathbb{R}^{d}}\left(\varrho\left(t+b_{n} z\right)-\varrho(t)\right) k(z) \mathrm{d} z\right)^{2} \mathrm{~d} t .
$$

Using Taylor's expansion of the product density $\varrho$ in $t=\left(t_{1}, \ldots, t_{d}\right)^{\prime}$ we get

$$
\varrho\left(t+b_{n} z\right)=\varrho(t)+b_{n} \sum_{i=1}^{d} z_{i} \frac{\partial}{\partial t_{i}} \varrho\left(t+\theta_{i} b_{n} z\right)
$$




$$
=\varrho(t)+b_{n} \sum_{i=1}^{d} z_{i} \frac{\partial}{\partial t_{i}} \varrho(t)+b_{n} \sum_{i=1}^{d} z_{i}\left(\frac{\partial}{\partial t_{i}} \varrho\left(t+\theta_{i} b_{n} z\right)-\frac{\partial}{\partial t_{i}} \varrho(t)\right)
$$

with $\theta_{i}=\theta_{i}(t) \in[0,1], i=1, \ldots, d$. The symmetry of the kernel function $k$ and the uniform Lipschitz-continuity of the first-order partial derivatives of the product density (with $L$ being the maximum of the Lipschitz constants) entail

$$
\begin{aligned}
\left|\int_{\mathbb{R}^{d}}\left(\varrho\left(t+b_{n} z\right)-\varrho(t)\right) k(z) \mathrm{d} z\right| & =b_{n}\left|\sum_{i=1}^{d} \int_{\mathbb{R}^{d}} z_{i}\left(\frac{\partial}{\partial t_{i}} \varrho\left(t+\theta_{i} b_{n} z\right)-\frac{\partial}{\partial t_{i}} \varrho(t)\right) k(z) \mathrm{d} z\right| \\
& \leq b_{n}^{2} L \sum_{i=1}^{d} \int_{\mathbb{R}^{d}}\left|z_{i}\right|^{2} k(z) \mathrm{d} z .
\end{aligned}
$$

This implies

$$
b_{n}^{d}\left|W_{n}\right| \int_{K}\left(\mathbb{E} \hat{\varrho}_{n}(t)-\lambda \varrho(t)\right)^{2} \mathrm{~d} t=\mathcal{O}\left(b_{n}^{d+4}\left|W_{n}\right|\right)
$$

as $n \rightarrow \infty$. Now we will prove the asymptotic representation

$$
b_{n}^{d}\left|W_{n}\right| \int_{K} \operatorname{Var}\left(\varrho_{n}(t)\right) \mathrm{d} t=\lambda \int_{K} \varrho(t) \mathrm{d} t \int_{\mathbb{R}^{d}} k^{2}(x) \mathrm{d} x+\mathcal{O}\left(b_{n}^{d \wedge 2}\right) .
$$

Using the representation (4.17) in Heinrich [10] we obtain

$$
\begin{aligned}
b_{n}^{2 d}\left|W_{n}\right|^{2} \operatorname{Var}\left(\hat{\varrho}_{n}(t)\right)= & \int_{\left(\mathbb{R}^{d}\right)^{2}} \mathbb{1}_{W_{n}}(x) k^{2}\left(\frac{y-x-t}{b_{n}}\right) \alpha^{(2)}(\mathrm{d}(x, y)) \\
& +\int_{\left(\mathbb{R}^{d}\right)^{2}} \mathbb{1}_{W_{n}}(x) \mathbb{1}_{W_{n}}(y) k\left(\frac{y-x-t}{b_{n}}\right) k\left(\frac{x-y-t}{b_{n}}\right) \alpha^{(2)}(\mathrm{d}(x, y)) \\
& +\int_{\left(\mathbb{R}^{d}\right)^{3}} \mathbb{1}_{W_{n}}(x) k\left(\frac{y-x-t}{b_{n}}\right) k\left(\frac{z-x-t}{b_{n}}\right) \alpha^{(3)}(\mathrm{d}(x, y, z)) \\
& +\int_{\left(\mathbb{R}^{d}\right)^{3}} \mathbb{1}_{W_{n}}(x) \mathbb{1}_{W_{n}}(y) k\left(\frac{y-x-t}{b_{n}}\right) k\left(\frac{z-y-t}{b_{n}}\right) \alpha^{(3)}(\mathrm{d}(x, y, z)) \\
& +\int_{\left(\mathbb{R}^{d}\right)^{3}} \mathbb{1}_{W_{n}}(x) \mathbb{1}_{W_{n}}(z) k\left(\frac{y-x-t}{b_{n}}\right) k\left(\frac{x-z-t}{b_{n}}\right) \alpha^{(3)}(\mathrm{d}(x, y, z)) \\
& +\int_{\left(\mathbb{R}^{d}\right)^{3}} \mathbb{1}_{W_{n}}(x) \mathbb{1}_{W_{n}}(z) k\left(\frac{y-x-t}{b_{n}}\right) k\left(\frac{y-z-t}{b_{n}}\right) \alpha^{(3)}(\mathrm{d}(x, y, z)) \\
& +\int_{\left(\mathbb{R}^{d}\right)^{4}} \mathbb{1}_{W_{n}}(x) \mathbb{1}_{W_{n}}(z) k\left(\frac{y-x-t}{b_{n}}\right) k\left(\frac{v-z-t}{b_{n}}\right) \\
& {\left[\alpha^{(4)}(\mathrm{d}(x, y, z, v))-\alpha^{(2)}(\mathrm{d}(x, y)) \alpha^{(2)}(\mathrm{d}(z, v))\right], }
\end{aligned}
$$

where 


$$
\begin{aligned}
& \alpha^{(4)}(\mathrm{d}(x, y, z, v))-\alpha^{(2)}(\mathrm{d}(x, y)) \alpha^{(2)}(\mathrm{d}(z, v)) \\
& =\quad \gamma^{(4)}(\mathrm{d}(x, y, z, v))+\gamma^{(1)}(\mathrm{d} x) \gamma^{(3)}(\mathrm{d}(y, z, v))+\gamma^{(1)}(\mathrm{d} y) \gamma^{(3)}(\mathrm{d}(x, z, v)) \\
& \quad+\gamma^{(1)}(\mathrm{d} z) \gamma^{(3)}(\mathrm{d}(x, y, v))+\gamma^{(1)}(\mathrm{d} v) \gamma^{(3)}(\mathrm{d}(x, y, z))+\gamma^{(2)}(\mathrm{d}(x, z)) \gamma^{(2)}(\mathrm{d}(y, v)) \\
& \quad+\gamma^{(2)}(\mathrm{d}(x, v)) \gamma^{(2)}(\mathrm{d}(y, z))+\gamma^{(2)}(\mathrm{d}(x, z)) \gamma^{(1)}(\mathrm{d} y) \gamma^{(1)}(\mathrm{d} v)+\gamma^{(2)}(\mathrm{d}(x, v)) \gamma^{(1)}(\mathrm{d} y) \gamma^{(1)}(\mathrm{d} z) \\
& \quad+\gamma^{(2)}(\mathrm{d}(y, z)) \gamma^{(1)}(\mathrm{d} x) \gamma^{(1)}(\mathrm{d} v)+\gamma^{(2)}(\mathrm{d}(y, v)) \gamma^{(1)}(\mathrm{d} x) \gamma^{(1)}(\mathrm{d} z) .
\end{aligned}
$$

First we consider the two integrals with respect to the second-order factorial moment measure $\alpha^{(2)}$. For the first one we obtain

$$
\begin{aligned}
\frac{1}{b_{n}^{d}\left|W_{n}\right|} \int_{K} \int_{\left(\mathbb{R}^{d}\right)^{2}} \mathbb{1}_{W_{n}}(x) k^{2}\left(\frac{y-x-t}{b_{n}}\right) \alpha^{(2)}(\mathrm{d}(x, y)) \mathrm{d} t & =\lambda \int_{K} \int_{\mathbb{R}^{d}} k^{2}(y) \varrho\left(b_{n} y+t\right) \mathrm{d} y \mathrm{~d} t \\
& =\lambda \int_{K} \varrho(t) \mathrm{d} t \int_{\mathbb{R}^{d}} k^{2}(y) \mathrm{d} y+\mathcal{O}\left(b_{n}^{2}\right)
\end{aligned}
$$

as $n \rightarrow \infty$ which can be seen by Taylor's expansion of $\varrho\left(b_{n} y+t\right)$ in $t$, using the uniform Lipschitzcontinuity of the first-order partial derivatives of the product density, and the boundedness and the symmetry of the kernel function $k$ which entails boundedness and symmetry of $k^{2}$. For the second integral with respect to the second-order factorial moment measure $\alpha^{(2)}$ we have

$$
\begin{aligned}
& \frac{1}{b_{n}^{d}\left|W_{n}\right|} \int_{K} \int_{\left.\mathbb{R}^{d}\right)^{2}} \mathbb{1}_{W_{n}}(x) \mathbb{1}_{W_{n}}(y) k\left(\frac{y-x-t}{b_{n}}\right) k\left(\frac{x-y-t}{b_{n}}\right) \alpha^{(2)}(\mathrm{d}(x, y)) \mathrm{d} t \\
& =b_{n}^{d} \lambda \int_{\mathbb{R}^{d}} \int_{\mathbb{R}^{d}} \frac{\left|W_{n} \cap\left(W_{n}-b_{n} y-b_{n} t\right)\right|}{\left|W_{n}\right|} k(y) k(y-2 t) \varrho\left(b_{n} y+b_{n} t\right) \mathbb{1}_{K}\left(b_{n} t\right) \mathrm{d} y \mathrm{~d} t \\
& =\mathcal{O}\left(b_{n}^{d}\right)
\end{aligned}
$$

as $n \rightarrow \infty$ due to the continuity of the product density $\varrho$ in $K \oplus b(o, \varepsilon)$ for some $\varepsilon>0$ if $o \in K \oplus b(o, \varepsilon)$ (otherwise the integral vanishes eventually).

Now we consider the integrals with respect to the third-order factorial moment measure $\alpha^{(3)}$. For the first of these integrals we have

$$
\begin{aligned}
& \frac{1}{b_{n}^{d}\left|W_{n}\right|} \int_{\left(\mathbb{R}^{d}\right)^{4}} \mathbb{1}_{K}(t) \mathbb{1}_{W_{n}}(x) k\left(\frac{y-x-t}{b_{n}}\right) k\left(\frac{z-x-t}{b_{n}}\right) \mathrm{d} t \alpha^{(3)}(\mathrm{d}(x, y, z)) \\
& =\frac{1}{b_{n}^{d}\left|W_{n}\right|} \int_{\left(\mathbb{R}^{d}\right)^{4}} \mathbb{1}_{K}(t) \mathbb{1}_{W_{n}}(x) k\left(\frac{y-x-t}{b_{n}}\right) k\left(\frac{z-x-t}{b_{n}}\right) \mathrm{d} t\left[\gamma^{(3)}(\mathrm{d}(x, y, z))+\right. \\
& =b_{n}^{d} \lambda \int_{\left(\mathbb{R}^{d}\right)^{3}} \mathbb{1}_{K}(t) k(y) k(z) c^{(2)}\left(b_{n} y+t, b_{n} z+t\right) \mathrm{d} y \mathrm{~d} z \mathrm{~d} t \\
& +b_{n}^{d} \lambda^{2} \int_{\left(\mathbb{R}^{d}\right)^{3}} \mathbb{1}_{K}(t) k(y) k(y+z) c^{(2)}\left(b_{n} z\right) \mathrm{d} y \mathrm{~d} z \mathrm{~d} t \\
& +2 b_{n}^{d} \lambda^{2} \int_{\left(\mathbb{R}^{d}\right)^{3}} \mathbb{1}_{K}(t) k(y) k(z) c^{(2)}\left(b_{n} z+t\right) \mathrm{d} y \mathrm{~d} z \mathrm{~d} t
\end{aligned}
$$




$$
\begin{aligned}
& +b_{n}^{d} \lambda^{3} \int_{\left(\mathbb{R}^{d}\right)^{3}} \mathbb{1}_{K}(t) k(y) k(z) \mathrm{d} y \mathrm{~d} z \mathrm{~d} t \\
= & \mathcal{O}\left(b_{n}^{d}\right)
\end{aligned}
$$

as $n \rightarrow \infty$. For deriving this asymptotic order we have used Lebesgue's dominated convergence theorem which is applicable due to $|K|<\infty$, the boundedness assumptions on the kernel function, the continuity of $c^{(2)}$, and $\sup _{u, v \in K \oplus b(o, \varepsilon)}\left|c^{(3)}(u, v)\right|<\infty$ for some $\varepsilon>0$. By analogous arguments we can show the asymptotic order of the other integrals with respect to the third-order factorial moment measure $\alpha^{(3)}$ to be $\mathcal{O}\left(b_{n}^{d}\right)$, too.

Let us now consider the integrals with respect to the factorial cumulant measures. Due to the finiteness of the total variations of order two and three the asymptotic order of the integrals with respect to $\gamma^{(2)}$ and $\gamma^{(3)}$ is $\mathcal{O}\left(b_{n}^{d}\right)$. The integral with respect to $\gamma^{(4)}$ is

$$
\begin{aligned}
& \frac{1}{b_{n}^{d}\left|W_{n}\right|} \int_{\left(\mathbb{R}^{d}\right)^{5}} \mathbb{1}_{K}(t) \mathbb{1}_{W_{n}}(x) \mathbb{1}_{W_{n}}(z) k\left(\frac{y-x-t}{b_{n}}\right) k\left(\frac{v-z-t}{b_{n}}\right) \gamma^{(4)}(\mathrm{d}(x, y, z, v)) \mathrm{d} t \\
& =b_{n}^{d} \lambda \int_{\left(\mathbb{R}^{d}\right)^{4}} \frac{\left|W_{n} \cap\left(W_{n}-z\right)\right|}{\left|W_{n}\right|} \mathbb{1}_{K}(t) k(y) k(v) c^{(4)}\left(b_{n} y+t, z, b_{n} v+z+t\right) \mathrm{d} y \mathrm{~d} z \mathrm{~d} v \mathrm{~d} t .
\end{aligned}
$$

Due to $\sup _{u, v \in K \oplus b(o, \varepsilon)} \int_{\mathbb{R}^{d}}\left|c^{(4)}(u, w, v+w)\right| \mathrm{d} w<\infty$ for some $\varepsilon>0$ we find that this integral is of asymptotic order $\mathcal{O}\left(b_{n}^{d}\right)$. This completes the proof.

Lemma 5.1 will show that the $k$ th cumulant of certain random variables (including the ISE $I_{n}(K)$ ) is a sum of integrals that are indecomposable, in the sense that they cannot be represented as a product of two integrals. The rigorous definition of decomposability is as follows.

Let $f_{i}:\left(\mathbb{R}^{d}\right)^{p_{i}} \rightarrow \mathbb{R}$ be fixed measurable functions, let $k \in \mathbb{N}$ and $p_{i} \in \mathbb{N}$ with $i \in I=\{1, \ldots, k\}$ be fixed and set

$$
\Psi^{\left(p_{i}\right)}\left(f_{i}\right):=\sum_{x_{1}, \ldots, x_{p_{i}} \in \Psi} f_{i}\left(x_{1}, \ldots, x_{p_{i}}\right)
$$

Let $\mathbb{E}\left[\left|\Psi^{\left(p_{i}\right)}\left(f_{i}\right)\right|^{k}\right]<\infty$ for all $i \in I$. We will now find a representation of the mixed moment $M\left(\Psi^{\left(p_{1}\right)}\left(f_{1}\right), \ldots, \Psi^{\left(p_{k}\right)}\left(f_{k}\right)\right):=\mathbb{E}\left[\prod_{i=1}^{k} \Psi^{\left(p_{i}\right)}\left(f_{i}\right)\right]$ as a sum of integrals defined as follows.

For arbitrary $T \subseteq I, q \in\left\{1, \ldots, p_{T}\right\}$ with $p_{T}:=\sum_{i \in T} p_{i}, r \in\{1, \ldots, q\}$, and decompositions $\mathcal{P}_{T}=\left\{P_{1}, \ldots, P_{q}\right\}$ of $\left\{1, \ldots, p_{T}\right\}$ and $\mathcal{Q}=\left\{Q_{1}, \ldots, Q_{r}\right\}$ of $\{1, \ldots, q\}$ we define the integral

$$
\begin{aligned}
& I_{\mathcal{P}_{T}, \mathcal{Q}}\left(f_{i}: \quad i \in T\right) \\
& :=\int_{\left(\mathbb{R}^{d}\right)^{q}} \prod_{b=1}^{q} \prod_{a \in P_{b}} \mathbb{1}_{\left\{x_{a}=z_{b}\right\}} f_{i_{1}}\left(x_{1}, \ldots, x_{p_{i_{1}}}\right) \\
& \quad \quad \times f_{i_{2}}\left(x_{p_{i_{1}}+1}, \ldots, x_{p_{i_{1}}+p_{i_{2}}}\right) \cdot \ldots \cdot f_{i_{\# T}}\left(x_{\sum_{j=1}^{\# T-1} p_{i_{j}}+1}, \ldots, x_{p_{T}}\right) \prod_{c=1}^{r} \gamma^{\left(\# Q_{c}\right)}\left(\mathrm{d} \mathbf{z}_{Q_{c}}\right),
\end{aligned}
$$

where $\left\{i_{1}, \ldots, i_{\# T}\right\}=T$ with $1 \leq i_{1}<i_{2}<\ldots<i_{\# T} \leq k$ and $\mathbf{z}_{Q_{c}}=\left(z_{q}\right)_{q \in Q_{c}}$. The elements of a set $P_{b}$ are the indices of the arguments of the functions $f_{i_{1}}, \ldots, f_{i_{\# T}}$ that are identical and 
distinct from all the arguments in every other set $P_{c} \neq P_{b}$. In the above-mentioned integral this is indicated by the term $\prod_{b=1}^{q} \prod_{a \in P_{b}} \mathbb{1}_{\left\{x_{a}=z_{b}\right\}}$. For the special case $T=I$ we have

$$
\begin{aligned}
& I_{\mathcal{P}_{I}, \mathcal{Q}}\left(f_{1}, \ldots, f_{k}\right) \\
& \quad=\int_{\left(\mathbb{R}^{d}\right)^{q}} \prod_{b=1}^{q} \prod_{a \in P_{b}} \mathbb{1}_{\left\{x_{a}=z_{b}\right\}} f_{1}\left(x_{1}, \ldots, x_{p_{1}}\right) \cdot \ldots \cdot f_{k}\left(x_{\sum_{i=1}^{k-1} p_{i}+1}, \ldots, x_{p_{I}}\right) \prod_{c=1}^{r} \gamma^{\left(\# Q_{c}\right)}\left(\mathrm{d} \mathbf{z}_{Q_{c}}\right) .
\end{aligned}
$$

Now the mixed moment $M\left(\Psi^{\left(p_{1}\right)}\left(f_{1}\right), \ldots, \Psi^{\left(p_{k}\right)}\left(f_{k}\right)\right)$ can be represented as

$$
\begin{aligned}
& M\left(\Psi^{\left(p_{1}\right)}\left(f_{1}\right), \ldots, \Psi^{\left(p_{k}\right)}\left(f_{k}\right)\right) \\
& =\sum_{q=1}^{p_{I}} \sum_{\substack{P_{1} \cup \ldots \cup P_{q} \\
=\left\{1, \ldots, p_{I}\right\}}} \int_{\left.\mathbb{R}^{d}\right)^{q}} \prod_{b=1}^{q} \prod_{a \in P_{b}} \mathbb{1}_{\left\{x_{a}=z_{b}\right\}} \\
& \times f_{1}\left(x_{1}, \ldots, x_{p_{1}}\right) \cdot \ldots \cdot f_{k}\left(x_{\sum_{i=1}^{k-1} p_{i}}, \ldots, x_{p_{I}}\right) \alpha^{(q)}\left(\mathrm{d}\left(z_{1}, \ldots, z_{q}\right)\right) \\
& =\sum_{q=1}^{p_{I}} \sum_{\substack{P_{1} \cup \ldots \cup P_{q} \\
=\{1, \ldots, p\}}} \sum_{r=1}^{q} \sum_{\substack{Q_{1} \cup \ldots \cup Q_{r} \\
=\{1, \ldots, q\}}} \int_{\left(\mathbb{R}^{d}\right)^{q}} \prod_{b=1}^{q} \prod_{a \in P_{b}} \mathbb{1}_{\left\{x_{a}=z_{b}\right\}} \\
& \times f_{1}\left(x_{1}, \ldots, x_{p_{1}}\right) \cdot \ldots \cdot f_{k}\left(x_{\sum_{i=1}^{k-1} p_{i}+1}, \ldots, x_{p_{I}}\right) \prod_{c=1}^{r} \gamma^{\left(\# Q_{c}\right)}\left(\mathrm{d} \mathbf{z}_{Q_{c}}\right),
\end{aligned}
$$

see Krickeberg [17]. With the above notation we have

$$
M\left(\Psi^{\left(p_{1}\right)}\left(f_{1}\right), \ldots, \Psi^{\left(p_{k}\right)}\left(f_{k}\right)\right)=\sum_{q=1}^{p_{I}} \sum_{\substack{\left.P_{1} \cup \ldots \cup P_{q}\right\} \\=\left\{1, \ldots, p_{I}\right\}}} \sum_{r=1}^{q} \sum_{\substack{Q_{1} \cup \ldots \cup Q_{r} \\=\{1, \ldots, q\}}} I_{\mathcal{P}_{I}, \mathcal{Q}}\left(f_{1}, \ldots, f_{k}\right) .
$$

Let $T=\left\{T_{1}, T_{2}\right\}$ be a decomposition of $I=\{1, \ldots, k\}$. An integral $I_{\mathcal{P}_{I}, \mathcal{Q}}\left(f_{1}, \ldots, f_{k}\right)$ is decomposable with respect to the decomposition $T=\left\{T_{1}, T_{2}\right\}$ if there exist a decomposition $\mathcal{P}^{(1)}$ of $\left\{1, \ldots, p_{T_{1}}\right\}$, a decomposition $\mathcal{P}^{(2)}$ of $\left\{1, \ldots, p_{T_{2}}\right\}, q_{1} \in\left\{1, \ldots, p_{T_{1}}\right\}$ and $q_{2} \in\left\{1, \ldots, p_{T_{2}}\right\}$ with $q_{1}+q_{2}=q$, and decompositions $\mathcal{Q}^{(1)}$ of $\left\{1, \ldots, q_{1}\right\}$ and $\mathcal{Q}^{(2)}$ of $\left\{1, \ldots, q_{2}\right\}$ such that

$$
I_{\mathcal{P}_{I}, \mathcal{Q}}\left(f_{1}, \ldots, f_{k}\right)=I_{\mathcal{P}_{T_{1}}, \mathcal{Q}^{(1)}}\left(f_{i}: i \in T_{1}\right) \cdot I_{\mathcal{P}_{T_{1}}, \mathcal{Q}^{(2)}}\left(f_{i}: i \in T_{2}\right) .
$$

An integral is called decomposable if there exists a nontrivial decomposition of $I$ such that this integral is decomposable with respect to this decomposition. An integral which is not decomposable with respect to any nontrivial decomposition is called indecomposable.

The following lemma is the key tool for the proof of the CLT for the ISE $I_{n}(K)$. It gives a representation of the $k$ th cumulant of certain random variables as a sum of indecomposable integrals. Let $\Gamma_{k}(X)$ denote the $k$ th cumulant of a real-valued random variable $X$ and $\operatorname{Cum}_{k}\left(X_{1}, \ldots, X_{k}\right)$ denote the mixed cumulant of a random vector $X=\left(X_{1}, \ldots, X_{k}\right)^{\prime} \in \mathbb{R}^{k}, k \geq 1$.

Lemma 5.1. Let $\Psi \sim P$ be a $P P$ in $\mathbb{R}^{d}$. Let $j, k \in \mathbb{N}$ be fixed, let $C_{i} \in \mathbb{R}$ be constants for $i=1, \ldots, j$, and set

$$
\Psi^{\left(p_{i}\right)}\left(f_{i}\right)=\sum_{x_{1}, \ldots, x_{p_{i}} \in \Psi} f_{i}\left(x_{1}, \ldots, x_{p_{i}}\right),
$$


where $f_{i}:\left(\mathbb{R}^{d}\right)^{p_{i}} \rightarrow \mathbb{R}$ is a fixed measurable function with $p_{i} \in \mathbb{N}$, for $i=1, \ldots, j$. Let $\mathbb{E}\left[\left|\Psi^{\left(p_{i}\right)}\left(f_{i}\right)\right|^{k}\right]<\infty$ for all $i=1, \ldots, j$. Then we have

$$
\Gamma_{k}\left(\sum_{i=1}^{j} C_{i} \Psi^{\left(p_{i}\right)}\left(f_{i}\right)\right)=\sum_{\substack{k_{1}+\ldots+k_{j}=k \\ k_{1}, \ldots, k_{j} \geq 0}} \frac{k !}{k_{1} ! \cdot \ldots \cdot k_{j} !} C_{1}^{k_{1}} \cdot \ldots \cdot C_{j}^{k_{j}} \mu_{k_{1}, \ldots, k_{j}}^{*},
$$

where

$$
\mu_{k_{1}, \ldots, k_{j}}^{*}:=\left(\sum_{q=1}^{p_{k_{1}, \ldots, k_{j}}} \sum_{\substack{P_{1} \cup \ldots \cup P_{q} \\=\left\{1, \ldots, p_{\left.k_{1}, \ldots, k_{j}\right\}}\right.}}^{q} \sum_{r=1}^{q} \sum_{\substack{Q_{1} \cup \ldots \cup Q_{r} \\=\{1, \ldots, q\}}}\right)^{*} I_{\mathcal{P}_{I}, \mathcal{Q}}(\underbrace{f_{1}, \ldots, f_{1}}_{k_{1}}, \ldots, \underbrace{f_{j}, \ldots, f_{j}}_{k_{j}})
$$

and $p_{k_{1}, \ldots, k_{j}}=\sum_{i=1}^{j} p_{i} k_{i}$. The summation $(\cdot)^{*}$ is taken only over the indecomposable integrals.

Proof. Due to multilinearity, symmetry, and homogeneity of the mixed cumulants we have

$$
\begin{aligned}
& \Gamma_{k}\left(\sum_{i=1}^{j} C_{i} \Psi^{\left(p_{i}\right)}\left(f_{i}\right)\right)=\operatorname{Cum}_{k}\left(\sum_{i=1}^{j} C_{i} \Psi_{f_{i}}, \ldots, \sum_{i=1}^{j} C_{i} \Psi^{\left(p_{i}\right)}\left(f_{i}\right)\right) \\
&=\sum_{\substack{k_{1}+\ldots+k_{j}=k \\
k_{1}, \ldots, k_{j} \geq 0}} \frac{k !}{k_{1} ! \cdot \ldots \cdot k_{j} !} C_{1}^{k_{1}} \cdot \ldots \cdot C_{j}^{k_{j}} \\
& \times \operatorname{Cum}_{k}(\underbrace{\Psi^{\left(p_{1}\right)}\left(f_{1}\right), \ldots, \Psi^{\left(p_{1}\right)}\left(f_{1}\right)}_{k_{1}}, \ldots, \underbrace{\Psi^{\left(p_{j}\right)}\left(f_{j}\right), \ldots, \Psi^{\left(p_{j}\right)}\left(f_{j}\right)}_{k_{j}}) .
\end{aligned}
$$

In order to prove the identity

$$
\mu_{k_{1}, \ldots, k_{j}}^{*}=\operatorname{Cum}_{k}(\underbrace{\Psi^{\left(p_{1}\right)}\left(f_{1}\right), \ldots, \Psi^{\left(p_{1}\right)}\left(f_{1}\right)}_{k_{1}}, \ldots, \underbrace{\Psi^{\left(p_{j}\right)}\left(f_{j}\right), \ldots, \Psi^{\left(p_{j}\right)}\left(f_{j}\right)}_{k_{j}})
$$

for all $k_{1}, \ldots, k_{j} \in\{0, \ldots, k\}$ with $\sum_{i=1}^{j} k_{i}=k$ we will proceed as in Jolivet [15] and Leonov and Shiryaev [18]. Let $k_{1}, \ldots, k_{j} \in\{0, \ldots, k\}$ with $\sum_{i=1}^{j} k_{i}=k$ and set $\Psi_{i}=\Psi^{\left(p_{i}\right)}\left(g_{i}\right)$, where

$$
g_{i}= \begin{cases}f_{1} & \text { for } i \in\left\{1, \ldots, k_{1}\right\} \\ f_{2} & \text { for } i \in\left\{k_{1}+1, \ldots, k_{1}+k_{2}\right\} \\ & \vdots \\ f_{j} & \text { for } i \in\left\{k_{1}+\ldots+k_{j-1}+1, \ldots, k\right\} .\end{cases}
$$

Then we have

$$
\left(\begin{array}{c}
\Psi_{1} \\
\vdots \\
\Psi_{k_{1}} \\
\Psi_{k_{1}+1} \\
\vdots \\
\Psi_{k}
\end{array}\right)=\left(\begin{array}{c}
\Psi^{\left(p_{1}\right)}\left(f_{1}\right) \\
\vdots \\
\Psi^{\left(p_{1}\right)}\left(f_{1}\right) \\
\Psi^{\left(p_{2}\right)}\left(f_{2}\right) \\
\vdots \\
\Psi^{\left(p_{j}\right)}\left(f_{j}\right)
\end{array}\right)
$$


With $M\left(\Psi_{1}, \ldots, \Psi_{k}\right)=\mathbb{E}\left[\prod_{i=1}^{k} \Psi_{i}\right]$ and $I=\{1, \ldots, k\}$ we have

$$
\begin{aligned}
\operatorname{Cum}_{k}\left(\Psi_{1}, \ldots, \Psi_{k}\right) & =M\left(\Psi_{1}, \ldots, \Psi_{k}\right)-\sum_{j=2}^{k} \sum_{I_{1} \cup \ldots \cup I_{j}=I} \prod_{i=1}^{j} \operatorname{Cum}_{\# I_{i}}\left(\Psi_{a}: a \in I_{i}\right) \\
& =\Sigma_{\text {indec }}+\Sigma_{\text {dec }}-\mathcal{C}
\end{aligned}
$$

(see Leonov and Shiryaev [18]), where $\Sigma_{\text {dec }}$ is the sum over the decomposable integrals from $M\left(\Psi_{1}, \ldots, \Psi_{k}\right)$,

$$
\mu_{k_{1}, \ldots, k_{j}}^{*} \equiv \Sigma_{\text {indec }}=M\left(\Psi_{1}, \ldots, \Psi_{k}\right)-\Sigma_{\text {dec }}
$$

is the sum over all indecomposable integrals from $M\left(\Psi_{1}, \ldots, \Psi_{k}\right)$, and

$$
\mathcal{C}=\sum_{j=2}^{k} \sum_{I_{1} \cup \ldots \cup I_{j}=I} \prod_{i=1}^{j} \operatorname{Cum}_{\# I_{i}}\left(\Psi_{a}: a \in I_{i}\right)
$$

denotes the remaining term.

For $j \in\{2, \ldots, k\}$ and a fixed decomposition $\left\{I_{1}, \ldots, I_{j}\right\}$ of $I=\{1, \ldots, k\}$, a summand $\prod_{i=1}^{j} \operatorname{Cum}_{\# I_{i}}\left(\Psi_{a}: a \in I_{i}\right)$ of $\mathcal{C}$ factorizes with respect to a decomposition $T=\left\{T_{1}, T_{2}\right\}$ if for each $i \in\{1, \ldots, j\}$ we have either $I_{i} \subseteq T_{1}$ or $I_{i} \subseteq T_{2}$, that is, if the summand can be written as

$$
\prod_{i=1}^{j} \operatorname{Cum}_{\# I_{i}}\left(\Psi_{a}: a \in I_{i}\right)=\prod_{\substack{i=1 \\ I_{i} \subseteq T_{1}}}^{j} \operatorname{Cum}_{\# I_{i}}\left(\Psi_{a}: a \in I_{i}\right) \cdot \prod_{\substack{i=1 \\ I_{i} \subseteq T_{2}}}^{j} \operatorname{Cum}_{\# I_{i}}\left(\Psi_{a}: a \in I_{i}\right) .
$$

Note that due to $j \geq 2$ each summand $\prod_{i=1}^{j} \operatorname{Cum}_{\# I_{i}}\left(\Psi_{a}: a \in I_{i}\right)$ factorizes with respect to at least one nontrivial decomposition.

Let $P_{I}$ be the distribution of the vector $\left(\Psi_{1}, \ldots, \Psi_{k}\right)^{\prime}$ which is determined by the distribution $P$ of the PP $\Psi$. For all $S \subseteq I$, let $P_{S}$ be the distribution of the vector $\left(\Psi_{a}\right)_{a \in S}$. Every term in $\mathcal{C}$ that factorizes with respect to a fixed decomposition $T=\left\{T_{1}, T_{2}\right\}$ of $I$ is completely determined by the marginals $P_{T_{1}}$ and $P_{T_{2}}$. The same is true for every term in $\Sigma_{\text {dec }}$ that is decomposable with respect to $T$.

Let $T^{(1)}=\left\{T_{1}^{(1)}, T_{2}^{(1)}\right\}$ be an arbitrary fixed decomposition of $I$. The sum over the terms of $\Sigma_{\text {dec }}$ that are decomposable with respect to $T^{(1)}$ is denoted by $\Sigma_{\text {dec }}^{(1)}$, and the sum over the terms of $\mathcal{C}$ that factorize with respect to $T^{(1)}$ is denoted by $\mathcal{C}_{\text {dec }}^{(1)}$. Let

$$
\Sigma^{(1)}=\Sigma_{\mathrm{dec}}-\Sigma_{\mathrm{dec}}^{(1)}
$$

and

$$
\mathcal{C}^{(1)}=\mathcal{C}-\mathcal{C}_{\mathrm{dec}}^{(1)}
$$

Then we have

$$
\operatorname{Cum}_{k}\left(\Psi_{1}, \ldots, \Psi_{k}\right)=\Sigma_{\text {indec }}+\Sigma^{(1)}+\Sigma_{\text {dec }}^{(1)}-\mathcal{C}^{(1)}-\mathcal{C}_{\mathrm{dec}}^{(1)}
$$

Now we will show

$$
\Sigma_{\mathrm{dec}}^{(1)}=\mathcal{C}_{\mathrm{dec}}^{(1)}
$$


To this end we set $\tilde{P}_{I}:=P_{T_{1}^{(1)}} \otimes P_{T_{2}^{(1)}}$, where $P_{T_{1}^{(1)}}$ and $P_{T_{2}^{(1)}}$ are the distributions of the random vectors $\left(\Psi_{a}: a \in T_{1}^{(1)}\right)$ and $\left(\Psi_{a}: a \in T_{2}^{(1)}\right)$, respectively. Let $\left(\tilde{\Psi}_{1}, \ldots, \tilde{\Psi}_{k}\right)^{\prime}$ be a random vector with distribution $\tilde{P}_{I}$, and let $\tilde{\Sigma}_{\text {indec }}, \tilde{\Sigma}^{(1)}, \tilde{\Sigma}_{\text {dec }}^{(1)}, \tilde{\mathcal{C}}^{(1)}$, and $\tilde{\mathcal{C}}_{\text {dec }}^{(1)}$ be defined as $\Sigma_{\text {indec }}, \Sigma^{(1)}, \Sigma_{\text {dec }}^{(1)}$, $\mathcal{C}^{(1)}$, and $\mathcal{C}_{\text {dec }}^{(1)}$ above, with $\left(\Psi_{1}, \ldots, \Psi_{k}\right)^{\prime}$ replaced by $\left(\tilde{\Psi}_{1}, \ldots, \tilde{\Psi}_{k}\right)^{\prime}$. By construction we have $\left(\tilde{\Psi}_{a}: a \in T_{i}^{(1)}\right) \sim P_{T_{i}^{(1)}}$, that is, $\left(\tilde{\Psi}_{a}: a \in T_{i}^{(1)}\right) \stackrel{\mathrm{d}}{=}\left(\Psi_{a}: a \in T_{i}^{(1)}\right), i=1,2$. Hence the fact that $\Sigma_{\text {dec }}^{(1)}$ and $\mathcal{C}_{\text {dec }}^{(1)}$ are completely determined by the marginals $P_{T_{1}^{(1)}}$ and $P_{T_{2}^{(1)}}$ implies $\tilde{\Sigma}_{\text {dec }}^{(1)}=\Sigma_{\text {dec }}^{(1)}$ and $\tilde{\mathcal{C}}_{\mathrm{dec}}^{(1)}=\mathcal{C}_{\mathrm{dec}}^{(1)}$. In particular we have

$$
\operatorname{Cum}_{k}\left(\tilde{\Psi}_{1}, \ldots, \tilde{\Psi}_{k}\right)=\tilde{\Sigma}_{\mathrm{indec}}+\tilde{\Sigma}^{(1)}+\Sigma_{\mathrm{dec}}^{(1)}-\tilde{\mathcal{C}}^{(1)}-\mathcal{C}_{\mathrm{dec}}^{(1)}
$$

Clearly, $\left(\tilde{\Psi}_{a}: a \in T_{1}^{(1)}\right)$ and $\left(\tilde{\Psi}_{a}: a \in T_{2}^{(1)}\right)$ are independent by construction. This implies the left-hand side in (3) to be equal to zero. Since the mixed moment

$$
M\left(\tilde{\Psi}_{1}, \ldots, \tilde{\Psi}_{k}\right)=\mathbb{E}\left[\prod_{i=1}^{k} \tilde{\Psi}_{i}\right]=\mathbb{E}\left[\prod_{i \in T_{1}^{(1)}} \tilde{\Psi}_{i}\right] \cdot \mathbb{E}\left[\prod_{i \in T_{2}^{(1)}} \tilde{\Psi}_{i}\right]=\tilde{\Sigma}_{\mathrm{dec}}^{(1)}
$$

is decomposable with respect to the decomposition $T^{(1)}$ we also have $\tilde{\Sigma}_{\text {indec }}=0$ and $\tilde{\Sigma}^{(1)}=0$.

Finally the independence of $\left(\tilde{\Psi}_{a}: a \in T_{1}^{(1)}\right)$ and $\left(\tilde{\Psi}_{a}: a \in T_{2}^{(1)}\right)$ yields $\operatorname{Cum}_{k}\left(\tilde{\Psi}_{a}: a \in K\right)=0$ for all $K \subseteq\{1, \ldots, k\}$ with $K \cap T_{1}^{(1)} \neq \emptyset$ and $K \cap T_{2}^{(1)} \neq \emptyset$. Since every summand in $\tilde{\mathcal{C}}^{(1)}$ contains a factor of this type we obtain $\tilde{\mathcal{C}}^{(1)}=0$.

Altogether this proves (2) by equation (3). As a result we have

$$
\operatorname{Cum}_{k}\left(\Psi_{1}, \ldots, \Psi_{k}\right)=\Sigma_{\text {indec }}+\Sigma^{(1)}-\mathcal{C}^{(1)} .
$$

Now we go through all possible decompositions of $I$ in this manner. Since every term of $\Sigma_{\text {dec }}$ is decomposable with respect to some decomposition and every term of $\mathcal{C}$ factorizes with respect to some decomposition, this yields

$$
\Sigma_{\text {dec }}=\mathcal{C}
$$

and hence

$$
\operatorname{Cum}_{k}\left(\Psi_{1}, \ldots, \Psi_{k}\right)=\Sigma_{\text {indec }} .
$$

In summary we have

$$
\operatorname{Cum}_{k}(\underbrace{\Psi^{\left(p_{1}\right)}\left(f_{1}\right), \ldots, \Psi^{\left(p_{1}\right)}\left(f_{1}\right)}_{k_{1}}, \ldots, \underbrace{\Psi^{\left(p_{j}\right)}\left(f_{j}\right), \ldots, \Psi^{\left(p_{j}\right)}\left(f_{j}\right)}_{k_{j}})=\mu_{k_{1}, \ldots, k_{j}}^{*}
$$

for all $k_{1}, \ldots, k_{j} \in\{0, \ldots, k\}$ with $\sum_{i=1}^{j} k_{i}=k$. This completes the proof.

The cumulants of the ISE $I_{n}(K)$ can be represented by a sum of indecomposable and irreducible integrals which will be shown in Lemma 5.2. First we give a definition of an irreducible integral. This definition is closely related to the special form of the functions 


$$
\begin{aligned}
& f_{1}:\left(\mathbb{R}^{d}\right)^{4} \rightarrow \mathbb{R}, \\
& \quad\left(x_{1}, x_{2}, x_{3}, x_{4}\right) \mapsto \mathbb{1}_{W_{n}}\left(x_{1}\right) \mathbb{1}_{W_{n}}\left(x_{3}\right) \mathbb{1}_{\left\{x_{1} \neq x_{2}, x_{3} \neq x_{4}\right\}} \int_{K} k\left(\frac{x_{2}-x_{1}-t}{b_{n}}\right) k\left(\frac{x_{4}-x_{3}-t}{b_{n}}\right) \mathrm{d} t,
\end{aligned}
$$

and

$$
f_{2}:\left(\mathbb{R}^{d}\right)^{2} \rightarrow \mathbb{R}, \quad\left(x_{1}, x_{2}\right) \mapsto \mathbb{1}_{W_{n}}\left(x_{1}\right) \mathbb{1}_{\left\{x_{1} \neq x_{2}\right\}} \int_{K} k\left(\frac{x_{2}-x_{1}-t}{b_{n}}\right) \lambda \varrho(t) \mathrm{d} t .
$$

An integral $I_{\mathcal{P}, \mathcal{Q}}(\underbrace{f_{1}, \ldots, f_{1}}_{k-j}, \underbrace{f_{2}, \ldots, f_{2}}_{j}), j=0, \ldots, k-1$, with $\mathcal{P}=\left\{P_{1}, \ldots, P_{q}\right\}$ and $\mathcal{Q}=$ $\left\{Q_{1}, \ldots, Q_{r}\right\}$ (see page 11) is reducible if there are indices $a, b \in\{1, \ldots, q\}, c, d \in\{1, \ldots, r\}$, and an odd number $i \in\{1, \ldots, 4 k-4 j\}$ such that $P_{a}=\{i\}, P_{b}=\{i+1\}$, and $Q_{c}=\{a, b\}$ or $Q_{c}=\{a\}$ and $Q_{d}=\{b\}$. In other words, a reducible integral contains one of the terms

$$
\begin{aligned}
\int_{\left(\mathbb{R}^{d}\right)^{2}} f_{1}\left(x_{i}, x_{i+1}, x, y\right) \gamma^{(2)}\left(\mathrm{d}\left(x_{i}, x_{i+1}\right)\right), & \int_{\left(\mathbb{R}^{d}\right)^{2}} f_{1}\left(x, y, x_{i}, x_{i+1}\right) \gamma^{(2)}\left(\mathrm{d}\left(x_{i}, x_{i+1}\right)\right), \\
\int_{\left(\mathbb{R}^{d}\right)^{2}} f_{1}\left(x_{i}, x_{i+1}, x, y\right) \gamma^{(1)}\left(\mathrm{d} x_{i}\right) \gamma^{(1)}\left(\mathrm{d} x_{i+1}\right), & \quad \text { or } \int_{\left(\mathbb{R}^{d}\right)^{2}} f_{1}\left(x, y, x_{i}, x_{i+1}\right) \gamma^{(1)}\left(\mathrm{d} x_{i}\right) \gamma^{(1)}\left(\mathrm{d} x_{i+1}\right),
\end{aligned}
$$

with $x, y \notin\left\{x_{i}, x_{i+1}\right\}$, and the remaining functions contain neither $x_{i}$ nor $x_{i+1}$. We will call this term the reducible part of the integral. An integral can have more than one reducible part. An integral that is not reducible is called irreducible. For instance, the integral

$$
\begin{aligned}
& I_{\{\{1\},\{2\},\{3,5\},\{4,6\}\},\{\{1,2\},\{3,4\}\}}\left(f_{1}, f_{2}\right) \\
& \quad=\int_{\left(\mathbb{R}^{d}\right)^{2}\left(\mathbb{R}^{d}\right)^{2}} f_{1}\left(z_{1}, z_{2}, z_{3}, z_{4}\right) f_{2}\left(z_{3}, z_{4}\right) \gamma^{(2)}\left(\mathrm{d}\left(z_{1}, z_{2}\right)\right) \gamma^{(2)}\left(\mathrm{d}\left(z_{3}, z_{4}\right)\right)
\end{aligned}
$$

is reducible with reducible part $\int_{\left(\mathbb{R}^{d}\right)^{2}} f_{1}\left(z_{1}, z_{2}, z_{3}, z_{4}\right) \gamma^{(2)}\left(\mathrm{d}\left(z_{1}, z_{2}\right)\right)$, whereas the integral

$$
\begin{aligned}
& I_{\{\{1,5\},\{2\},\{3,6\},\{4\}\},\{\{1,2\},\{3,4\}\}}\left(f_{1}, f_{2}\right) \\
& \quad=\int_{\left(\mathbb{R}^{d}\right)^{2}\left(\mathbb{R}^{d}\right)^{2}} f_{1}\left(z_{1}, z_{2}, z_{3}, z_{4}\right) f_{2}\left(z_{1}, z_{3}\right) \gamma^{(2)}\left(\mathrm{d}\left(z_{1}, z_{2}\right)\right) \gamma^{(2)}\left(\mathrm{d}\left(z_{3}, z_{4}\right)\right)
\end{aligned}
$$

is irreducible.

Recall the sum of indecomposable integrals $\mu_{k-j, j}^{*}, j=0, \ldots, k$, see $(1)$. We denote the sum of irreducible integrals in $\mu_{k-j, j}^{*}$ by $\mu_{k-j, j}^{* *}, j=0, \ldots, k$. We will write $\mu_{k-(j+r), j+r}^{* * a}, a=1, \ldots, r$, for the term obtained from $\mu_{k-(j+r), j+r}^{* *}$ by replacing $a$ instances of $f_{2}$ with $\tilde{f}_{2}$, where the function $\tilde{f}_{2}$ is given by

$$
\tilde{f}_{2}(x, y):=\mathbb{1}_{W_{n}}(x) \int_{K}\left[k\left(\frac{y-x-t}{b_{n}}\right) \lambda\left(\int_{\mathbb{R}^{d}} R_{n}(z, t) k(z) \mathrm{d} z\right)\right] \mathrm{d} t
$$

with $R_{n}(z, t)=\sum_{i=1}^{d} z_{i}\left(\frac{\partial}{\partial t_{i}} \varrho\left(t+\theta_{i} b_{n} z\right)-\frac{\partial}{\partial t_{i}} \varrho(t)\right)$ and $\theta_{i}=\theta_{i}(t) \in[0,1], i=1, \ldots, d$. As a result, $\mu_{k-(j+r), j+r}^{* * a}$ contains only $j+r-a$ instances of $f_{2}$. 
Now we can state the lemma giving a representation of the $k$ th cumulant of the ISE in terms of indecomposable and irreducible integrals for $k \geq 2$.

Lemma 5.2. Let $k \geq 2$, and let $\Psi \sim P$ be a $\mathrm{B}_{4 k}$-mixing $P P$ in $\mathbb{R}^{d}$ with intensity $\lambda$ and product density $\varrho$. Let the first-order partial derivatives of the product density $\varrho$ be uniformly Lipschitzcontinuous in $K \oplus b(o, \varepsilon)$ for some $\varepsilon>0$. Let $k$ satisfy Condition $\mathcal{C}(d)($ iii).

Then the kth cumulant of the ISE $I_{n}(K)$ satisfies

$$
\Gamma_{k}\left(I_{n}(K)\right)=\sum_{j=0}^{k}\left(\begin{array}{c}
k \\
j
\end{array}\right) 2^{j} b_{n}^{j}\left(b_{n}^{d}\left|W_{n}\right|\right)^{j-2 k} \mu_{k-j, j}^{* * j} .
$$

Proof. In the first part of the proof we apply Lemma 5.1 in order to express the $k$ th cumulant by a sum of indecomposable integrals. Due to the smoothness conditions on the product density this representation can be further simplified. This is shown in the second part of the proof.

Due to the semi-invariance of the cumulants of order two and higher the $k$ th cumulant of $\int_{K}\left(\hat{\varrho}_{n}^{2}(t)-2 \lambda \varrho(t) \varrho_{n}(t)\right) \mathrm{d} t$ is identical to the $k$ th cumulant of $\left(I_{n}(K)-\mathbb{E} I_{n}(K)\right)$ for $k \geq 2$. Therefore we investigate the $k$ th cumulant of $\int_{K}\left(\hat{\varrho}_{n}^{2}(t)-2 \lambda \varrho(t) \varrho_{n}(t)\right) \mathrm{d} t$.

\section{Representation of the $k$ th cumulant by indecomposable integrals}

First we rewrite $\int_{K}\left(\hat{\varrho}_{n}^{2}(t)-2 \lambda \varrho(t) \hat{\varrho}_{n}(t)\right) \mathrm{d} t$. We have

$$
\begin{aligned}
\int_{K} & \left(\hat{\varrho}_{n}^{2}(t)-2 \lambda \varrho(t) \hat{\varrho}_{n}(t)\right) \mathrm{d} t \\
= & \sum_{\substack{x_{1}, x_{2}, x_{3}, x_{4} \in \Psi \\
x_{1} \neq x_{2}, x_{3} \neq x_{4}}}\left(b_{n}^{d}\left|W_{n}\right|\right)^{-2} \mathbb{1}_{W_{n}}\left(x_{1}\right) \mathbb{1}_{W_{n}}\left(x_{3}\right) \int_{K} k\left(\frac{x_{2}-x_{1}-t}{b_{n}}\right) k\left(\frac{x_{4}-x_{3}-t}{b_{n}}\right) \mathrm{d} t \\
& \quad-\sum_{x_{1}, x_{2} \in \Psi}^{*} 2\left(b_{n}^{d}\left|W_{n}\right|\right)^{-1} \mathbb{1}_{W_{n}}\left(x_{1}\right) \int_{K} k\left(\frac{x_{2}-x_{1}-t}{b_{n}}\right) \lambda \varrho(t) \mathrm{d} t \\
= & \sum_{x_{1}, x_{2}, x_{3}, x_{4} \in \Psi} C_{1} f_{1}\left(x_{1}, x_{2}, x_{3}, x_{4}\right)+\sum_{x_{1}, x_{2} \in \Psi} C_{2} f_{2}\left(x_{1}, x_{2}\right),
\end{aligned}
$$

with functions

$$
\begin{aligned}
& f_{1}:\left(\mathbb{R}^{d}\right)^{4} \rightarrow \mathbb{R}, \\
& \quad\left(x_{1}, x_{2}, x_{3}, x_{4}\right) \mapsto \mathbb{1}_{W_{n}}\left(x_{1}\right) \mathbb{1}_{W_{n}}\left(x_{3}\right) \mathbb{1}_{\left\{x_{1} \neq x_{2}, x_{3} \neq x_{4}\right\}} \int_{K} k\left(\frac{x_{2}-x_{1}-t}{b_{n}}\right) k\left(\frac{x_{4}-x_{3}-t}{b_{n}}\right) \mathrm{d} t,
\end{aligned}
$$

and

$$
f_{2}:\left(\mathbb{R}^{d}\right)^{2} \rightarrow \mathbb{R}, \quad\left(x_{1}, x_{2}\right) \mapsto \mathbb{1}_{W_{n}}\left(x_{1}\right) \mathbb{1}_{\left\{x_{1} \neq x_{2}\right\}} \int_{K} k\left(\frac{x_{2}-x_{1}-t}{b_{n}}\right) \lambda \varrho(t) \mathrm{d} t
$$


and constants $C_{1}:=\left(b_{n}^{d}\left|W_{n}\right|\right)^{-2}$ and $C_{2}:=-2\left(b_{n}^{d}\left|W_{n}\right|\right)^{-1}$. Since we have $|K|<\infty$ and since $k$ is bounded with bounded support the moments $\mathbb{E}\left[\left|\sum_{x_{1}, x_{2}, x_{3}, x_{4} \in \Psi} f_{1}\left(x_{1}, x_{2}, x_{3}, x_{4}\right)\right|^{k}\right]$ and $\mathbb{E}\left[\left|\sum_{x_{1}, x_{2} \in \Psi} f_{2}\left(x_{1}, x_{2}\right)\right|^{k}\right]$ are finite. Hence we can apply Lemma 5.1. Therefore the $k$ th cumulant $\Gamma_{k}\left(I_{n}(K)\right)$ of the ISE $I_{n}(K)$ satisfies

$$
\begin{aligned}
\Gamma_{k}\left(I_{n}(K)\right) & =\sum_{\substack{k_{1}+k_{2}=k \\
k_{1}, k_{2} \geq 0}} \frac{k !}{k_{1} ! k_{2} !}(-1)^{k_{2}} 2^{k_{2}}\left(b_{n}^{d}\left|W_{n}\right|\right)^{-2 k_{1}-k_{2}} \mu_{k_{1}, k_{2}}^{*} \\
& =\sum_{j=0}^{k}\left(\begin{array}{c}
k \\
j
\end{array}\right)(-1)^{j} 2^{j}\left(b_{n}^{d}\left|W_{n}\right|\right)^{j-2 k} \mu_{k-j, j}^{*} .
\end{aligned}
$$

\section{Representation of the cumulants by indecomposable and irreducible integrals}

The special form of the functions $f_{1}$ and $f_{2}$ allows a further simplification of the representation for the $k$ th cumulant given in Lemma 5.2. This simplification is based on the approximate identity

$$
\begin{aligned}
\int_{\left(\mathbb{R}^{d}\right)^{2}} f_{1}\left(x_{1}, x_{2}, x_{3}, x_{4}\right) \alpha^{(2)}\left(\mathrm{d}\left(x_{1}, x_{2}\right)\right) & =\int_{\left(\mathbb{R}^{d}\right)^{2}} f_{1}\left(x_{3}, x_{4}, x_{1}, x_{2}\right) \alpha^{(2)}\left(\mathrm{d}\left(x_{1}, x_{2}\right)\right) \\
& \approx b_{n}^{d}\left|W_{n}\right| f_{2}\left(x_{3}, x_{4}\right)
\end{aligned}
$$

for $x_{3}, x_{4} \notin\left\{x_{1}, x_{2}\right\}$, which implies the reducible integrals of $\mu_{k, 0}^{*}$ (except for the error terms) and integrals in $\mu_{k-\ell, \ell}^{*}, \ell=1, \ldots, k$, to cancel.

More precisely we start by combining two reducible integrals in $\mu_{k-j, j}^{*}, j=0, \ldots, k-1$. These integrals differ only by their reducible parts, in two possible ways. Either the two integrals' reducible parts are

$$
\int_{\left(\mathbb{R}^{d}\right)^{2}} f_{1}\left(x_{i}, x_{i+1}, x, y\right) \gamma^{(2)}\left(\mathrm{d}\left(x_{i}, x_{i+1}\right)\right) \quad \text { and } \quad \int_{\left(\mathbb{R}^{d}\right)^{2}} f_{1}\left(x_{i}, x_{i+1}, x, y\right) \gamma^{(1)}\left(\mathrm{d} x_{i}\right) \gamma^{(1)}\left(\mathrm{d} x_{i+1}\right)
$$

or they are

$$
\int_{\left(\mathbb{R}^{d}\right)^{2}} f_{1}\left(x, y, x_{i}, x_{i+1}\right) \gamma^{(2)}\left(\mathrm{d}\left(x_{i}, x_{i+1}\right)\right) \quad \text { and } \quad \int_{\left(\mathbb{R}^{d}\right)^{2}} f_{1}\left(x, y, x_{i}, x_{i+1}\right) \gamma^{(1)}\left(\mathrm{d} x_{i}\right) \gamma^{(1)}\left(\mathrm{d} x_{i+1}\right) .
$$

The sum of these two reducible integrals in $\mu_{k-j, j}^{*}$ is hence an integral which emerges from either of the two aforementioned integrals by replacing the respective reducible parts by

$$
\int_{\left(\mathbb{R}^{d}\right)^{2}} f_{1}\left(x_{i}, x_{i+1}, x, y\right) \alpha^{(2)}\left(\mathrm{d}\left(x_{i}, x_{i+1}\right)\right)
$$

or

$$
\int_{\left(\mathbb{R}^{d}\right)^{2}} f_{1}\left(x, y, x_{i}, x_{i+1}\right) \alpha^{(2)}\left(\mathrm{d}\left(x_{i}, x_{i+1}\right)\right),
$$

depending on the above distinction. If the integral has more than one reducible part, then we iterate the above procedure, eventually obtaining an irreducible integral. In the following, we will 
only consider irreducible integrals and integrals which arise from the above-mentioned combination and summation of reducible integrals. The latter integrals are also called reducible parts. Now we simplify one of the reducible parts (5) and (6) of a reducible integral by disintegration and Taylor's expansion, that is,

$$
\begin{aligned}
& \int_{\left(\mathbb{R}^{d}\right)^{2}} \mathbb{1}_{W_{n}}\left(x_{i}\right) \mathbb{1}_{W_{n}}(x) \int_{K} k\left(\frac{x_{i+1}-x_{i}-t}{b_{n}}\right) k\left(\frac{y-x-t}{b_{n}}\right) \mathrm{d} t \alpha^{(2)}\left(\mathrm{d}\left(x_{i}, x_{i+1}\right)\right) \\
& =b_{n}^{d}\left|W_{n}\right| \mathbb{1}_{W_{n}}(x) \int_{K} k\left(\frac{y-x-t}{b_{n}}\right) \lambda\left(\int_{\mathbb{R}^{d}} k\left(x_{i+1}\right) \varrho\left(b_{n} x_{i+1}+t\right) \mathrm{d} x_{i+1}\right) \mathrm{d} t \\
& =b_{n}^{d}\left|W_{n}\right| f_{2}(x, y)+b_{n} b_{n}^{d}\left|W_{n}\right| \tilde{f}_{2}(x, y),
\end{aligned}
$$

where

$$
\tilde{f}_{2}(x, y)=\mathbb{1}_{W_{n}}(x) \int_{K}\left[k\left(\frac{y-x-t}{b_{n}}\right) \lambda\left(\int_{\mathbb{R}^{d}} R_{n}(z, t) k(z) \mathrm{d} z\right)\right] \mathrm{d} t
$$

with $R_{n}(z, t)=\sum_{i=1}^{d} z_{i}\left(\frac{\partial}{\partial t_{i}} \varrho\left(t+\theta_{i} b_{n} z\right)-\frac{\partial}{\partial t_{i}} \varrho(t)\right)$ and $\theta_{i}=\theta_{i}(t) \in[0,1], i=1, \ldots, d$. Here we have used the symmetry of the kernel function $k$ so that only $\varrho(t)$ and the error term $R_{n}$ remain from Taylor's expansion. In the following we will refer to the above simplification by disintegration and Taylor's expansion as reduction of the integral. Note that the uniform Lipschitz-continuity of the first-order partial derivatives of the product density yields the upper bound

$$
\left|R_{n}(z, t)\right| \leq b_{n} L \sum_{i=1}^{d}\left|z_{i}\right|
$$

where $L$ is the maximum of the Lipschitz-constants.

An integral in $\mu_{k-j, j}^{*}$ is called $r$-reducible if it can be reduced exactly $r$ times (that is, if reduction as defined above can be applied exactly $r$ times), with $r \in\{0, \ldots, k-j\}$. Reducing an $r$-reducible integral $r$ times yields a sum of two parts. The first part is an integral in $\mu_{k-(j+r), j+r}^{* *}$ multiplied by $\left(b_{n}^{d}\left|W_{n}\right|\right)^{r}$ while the second part is a sum of integrals containing the error terms from all Taylor expansions performed in the reductions. Note that within this iterative scheme reductions can also be applied to error terms obtained from earlier reductions. We illustrate this procedure by an example involving three reductions of a 3-reducible integral in $\mu_{3,0}^{*}$ :

$$
\begin{aligned}
& \int_{\left(\mathbb{R}^{d}\right)^{8}} f_{1}\left(x_{1}, x_{2}, x_{3}, x_{4}\right) f_{1}\left(x_{1}, x_{2}, x_{5}, x_{6}\right) f_{1}\left(x_{1}, x_{2}, x_{7}, x_{8}\right) \\
& =b_{n}^{3 d}\left|W_{n}\right|^{3}\left(\int_{\left(\mathbb{R}^{d}\right)^{2}}\left(f_{2}\left(x_{1}, x_{2}\right)\right)^{3} \gamma^{(2)}\left(\mathrm{d}\left(x_{1}, x_{2}\right)\right)\right. \\
& +3 b_{n} \int_{\left(\mathbb{R}^{d}\right)^{4}}\left(f_{2}\left(x_{1}, x_{2}\right)\right)^{2} \tilde{f}_{2}\left(x_{1}, x_{2}\right) \gamma^{(2)}\left(\mathrm{d}\left(x_{1}, x_{2}\right)\right) \\
& +3 b_{n}^{2} \int_{\left(\mathbb{R}^{d}\right)^{4}} f_{2}\left(x_{1}, x_{2}\right)\left(\tilde{f}_{2}\left(x_{1}, x_{2}\right)\right)^{2} \gamma^{(2)}\left(\mathrm{d}\left(x_{1}, x_{2}\right)\right)
\end{aligned}
$$




$$
\left.+b_{n}^{3} \int_{\left(\mathbb{R}^{d}\right)^{4}}\left(\tilde{f}_{2}\left(x_{1}, x_{2}\right)\right)^{3} \gamma^{(2)}\left(\mathrm{d}\left(x_{1}, x_{2}\right)\right)\right)
$$

In the remaining terms $a$ instances of the function $f_{2}$ are replaced by $\tilde{f}_{2}, a=1, \ldots, r$. For each integral in $\mu_{k-(j+r), j+r}^{* * a}$ the number of $r$-reducible integrals in $\mu_{k-j, j}^{*}$ leading to this integral is $2^{r}\left(\begin{array}{c}k-j \\ r\end{array}\right)$. Hence we obtain the representation

$$
\mu_{k-j, j}^{*}=\sum_{r=0}^{k-j} 2^{r}\left(\begin{array}{c}
k-j \\
r
\end{array}\right)\left(b_{n}^{d}\left|W_{n}\right|\right)^{r} \sum_{a=0}^{r} b_{n}^{a}\left(\begin{array}{c}
r \\
a
\end{array}\right) \mu_{k-(j+r), j+r}^{* * a}
$$

for $j=0, \ldots, k$. The main terms are $\mu_{k-(j+r), j+r}^{* *} \equiv \mu_{k-(j+r), j+r}^{* * 0}, r=0, \ldots, k-j$, and the remaining terms are $\mu_{k-(j+r), j+r}^{* * a}, a=1, \ldots, r$. Equations (4) and (8) imply

$$
\begin{aligned}
\Gamma_{k}\left(I_{n}(K)\right) & =\sum_{i=0}^{k}(-1)^{i}\left(\begin{array}{c}
k \\
i
\end{array}\right) 2^{i}\left(b_{n}^{d}\left|W_{n}\right|\right)^{i-2 k} \mu_{k-i, i}^{*} \\
& =\sum_{i=0}^{k} \sum_{r=0}^{k-i} \sum_{a=0}^{r}(-1)^{i} \frac{k !}{i ! a !(r-a) !(k-(i+r)) !} 2^{i+r}\left(b_{n}^{d}\left|W_{n}\right|\right)^{i+r-2 k} b_{n}^{a} \mu_{k-(i+r), i+r}^{* * a} \\
& =\sum_{j=0}^{k} \sum_{i=0}^{j} \sum_{a=0}^{j-i}(-1)^{i} \frac{k !}{i ! a !(j-i-a) !(k-j) !} 2^{j}\left(b_{n}^{d}\left|W_{n}\right|\right)^{j-2 k} b_{n}^{a} \mu_{k-j, j}^{* * a} \\
& =\sum_{j=0}^{k}\left(\begin{array}{c}
k \\
j
\end{array}\right) 2^{j} b_{n}^{j}\left(b_{n}^{d}\left|W_{n}\right|\right)^{j-2 k} \mu_{k-j, j}^{* * j} .
\end{aligned}
$$

For the last line we consider the summands indexed by $j \in\{0, \ldots, k\}$. This yields

$$
\begin{aligned}
& \sum_{i=0}^{j} \sum_{a=0}^{j-i}(-1)^{i} \frac{k !}{i ! a !(j-i-a) !(k-j) !} 2^{j}\left(b_{n}^{d}\left|W_{n}\right|\right)^{j-2 k} b_{n}^{a} \mu_{k-j, j}^{* * a} \\
& \quad=\frac{k !}{(k-j) !} 2^{j}\left(b_{n}^{d}\left|W_{n}\right|\right)^{j-2 k} \sum_{a=0}^{j} \frac{1}{a !} b_{n}^{a} \mu_{k-j, j}^{* * a} \sum_{i=0}^{j-a}(-1)^{i} \frac{1}{i !(j-a-i) !} .
\end{aligned}
$$

Due to $\sum_{i=0}^{j-a}(-1)^{i} \frac{1}{i !(j-a-i) !}=0$ for $a=0, \ldots, j-1$ and $\sum_{i=0}^{0}(-1)^{i} \frac{1}{i !(j-i) !}=1$ the identity (9) follows and the proof is complete.

Now we are ready to prove the asymptotic representation of the variance of the ISE of the product density estimator.

Proof of Lemma 3.2. We use the representation of the second cumulant of the scaled ISE

$$
\Gamma_{2}\left(b_{n}^{d / 2}\left|W_{n}\right| I_{n}(K)\right)=b_{n}^{d}\left|W_{n}\right|^{2} \Gamma_{2}\left(I_{n}(K)\right)=\sum_{j=0}^{2}\left(\begin{array}{c}
2 \\
j
\end{array}\right) 2^{j} b_{n}^{j}\left(b_{n}^{d}\right)^{j-3}\left(\left|W_{n}\right|\right)^{j-2} \mu_{2-j, j}^{* * j}
$$


derived in Lemma 5.2. Now we will determine the asymptotic order of $b_{n}^{j}\left(b_{n}^{d}\right)^{j-3}\left(\left|W_{n}\right|\right)^{j-2} \mu_{2-j, j}^{* * j}$, $j=0,1,2$. The highest-order terms in $\mu_{2,0}^{* * 0}$ are

$$
\begin{gathered}
\int_{\left(\mathbb{R}^{d}\right)^{4}} f_{1}\left(x_{1}, x_{2}, x_{3}, x_{4}\right)\left[f_{1}\left(x_{1}, x_{2}, x_{3}, x_{4}\right)+f_{1}\left(x_{3}, x_{4}, x_{1}, x_{2}\right)\right. \\
\left.+f_{1}\left(x_{2}, x_{1}, x_{4}, x_{3}\right)+f_{1}\left(x_{4}, x_{3}, x_{2}, x_{1}\right)\right] \\
{\left[\gamma^{(2)}\left(\mathrm{d}\left(x_{1}, x_{2}\right)\right) \gamma^{(2)}\left(\mathrm{d}\left(x_{3}, x_{4}\right)\right)+\gamma^{(2)}\left(\mathrm{d}\left(x_{1}, x_{2}\right)\right) \gamma^{(2)}\left(\mathrm{d}\left(x_{3}, x_{4}\right)\right)\right.} \\
+\gamma^{(1)}\left(\mathrm{d} x_{1}\right) \gamma^{(1)}\left(\mathrm{d} x_{2}\right) \gamma^{(2)}\left(\mathrm{d}\left(x_{3}, x_{4}\right)\right)+\gamma^{(1)}\left(\mathrm{d} x_{3}\right) \gamma^{(1)}\left(\mathrm{d} x_{4}\right) \gamma^{(2)}\left(\mathrm{d}\left(x_{3}, x_{4}\right)\right) \\
\left.+\gamma^{(1)}\left(\mathrm{d} x_{1}\right) \gamma^{(1)}\left(\mathrm{d} x_{2}\right) \gamma^{(1)}\left(\mathrm{d} x_{3}\right) \gamma^{(1)}\left(\mathrm{d} x_{4}\right)\right] .
\end{gathered}
$$

Combining the factorial cumulant measures to factorial moment measures and multiplying with the scaling factor $\left(b_{n}^{3 d}\left|W_{n}\right|^{2}\right)^{-1}$ we obtain

$$
\begin{aligned}
\frac{1}{b_{n}^{3 d}\left|W_{n}\right|^{2}} \int_{\left(\mathbb{R}^{d}\right)^{4}} f_{1}\left(x_{1}, x_{2}, x_{3}, x_{4}\right)\left[f_{1}\left(x_{1}, x_{2}, x_{3}, x_{4}\right)+f_{1}\left(x_{3}, x_{4}, x_{1}, x_{2}\right)\right. & \left.\quad+f_{1}\left(x_{2}, x_{1}, x_{4}, x_{3}\right)+f_{1}\left(x_{4}, x_{3}, x_{2}, x_{1}\right)\right] \alpha^{(2)}\left(\mathrm{d}\left(x_{1}, x_{2}\right)\right) \alpha^{(2)}\left(\mathrm{d}\left(x_{3}, x_{4}\right)\right) \\
= & 2 \lambda^{2} \int_{\left(\mathbb{R}^{d}\right)^{2}} \mathbb{1}_{K}\left(b_{n} t_{1}+t_{2}\right) \mathbb{1}_{K}\left(t_{2}\right)\left(\int_{\mathbb{R}^{d}} k(x) k\left(x+t_{1}\right) \varrho\left(b_{n} x+b_{n} t_{1}+t_{2}\right) \mathrm{d} x\right)^{2} \mathrm{~d} t_{1} \mathrm{~d} t_{2} \\
+ & 2 \lambda^{2} \int_{\left(\mathbb{R}^{d}\right)^{2}} \mathbb{1}_{K}\left(b_{n} t_{1}-t_{2}\right) \mathbb{1}_{K}\left(t_{2}\right) \\
& \times\left(\int_{\mathbb{R}^{d}} \frac{\left|W_{n} \cap\left(W_{n}-b_{n} x+b_{n} t_{1}-t_{2}\right)\right|}{\left|W_{n}\right|} k(x) k\left(x+t_{1}\right) \varrho\left(b_{n} x+b_{n} t_{1}-t_{2}\right) \mathrm{d} x\right)^{2} \mathrm{~d} t_{1} \mathrm{~d} t_{2} \\
\underset{n \rightarrow \infty}{\longrightarrow} & 2 \lambda^{2} \int_{\mathbb{R}^{d}}(k * k)^{2}(t) \mathrm{d} t\left(\int_{K} \varrho^{2}(t) \mathrm{d} t+\int_{K \cap(-K)} \varrho^{2}(t) \mathrm{d} t\right) .
\end{aligned}
$$

The remaining part of $\mu_{2,0}^{* * 0}$, scaled with $\left(b_{n}^{3 d}\left|W_{n}\right|^{2}\right)^{-1}$, is of order $O\left(b_{n}^{d}+\left(b_{n}^{d}\left|W_{n}\right|\right)^{-1}\right)$ as $n \rightarrow \infty$. For integrals in $\mu_{2,0}^{* * 0}$ containing an integration with respect to $\gamma^{(5)}, \gamma^{(6)}, \gamma^{(7)}$ and $\gamma^{(8)}$, this is due to the finiteness of these measures' total variation. For the other integrals one uses the assumptions on the cumulant densities up to order four or the finiteness of the total variations $\left\|\gamma_{\text {red }}^{(k)}\right\|, k=2,3,4$. For example, if we do not assume the existence of the fourth-order cumulant density, the integral

$$
\begin{gathered}
\frac{1}{b_{n}^{3 d}\left|W_{n}\right|^{2}} \int_{\left(\mathbb{R}^{d}\right)^{8}} f_{1}\left(x_{1}, x_{2}, x_{3}, x_{4}\right) f_{1}\left(x_{5}, x_{6}, x_{7}, x_{8}\right) \gamma^{(4)}\left(\mathrm{d}\left(x_{1}, x_{2}, x_{5}, x_{6}\right)\right) \gamma^{(4)}\left(\mathrm{d}\left(x_{3}, x_{4}, x_{7}, x_{8}\right)\right) \\
=\frac{1}{b_{n}^{3 d}} \int_{\left(\mathbb{R}^{d}\right)^{8}} \frac{\left|W_{n} \cap\left(W_{n}-x_{5}\right)\right|\left|W_{n} \cap\left(W_{n}-x_{7}\right)\right|_{K}\left(t_{1}\right) \mathbb{1}_{K}\left(t_{2}\right)}{\left|W_{n}\right|^{2}} \\
\quad \times k\left(\frac{x_{2}-t_{1}}{b_{n}}\right) k\left(\frac{x_{4}-t_{1}}{b_{n}}\right) k\left(\frac{x_{6}-x_{5}-t_{2}}{b_{n}}\right) k\left(\frac{x_{8}-x_{7}-t_{2}}{b_{n}}\right) \\
\mathrm{d} t_{1} \mathrm{~d} t_{2} \gamma_{\text {red }}^{(4)}\left(\mathrm{d}\left(x_{2}, x_{5}, x_{6}\right)\right) \gamma_{\text {red }}^{(4)}\left(\mathrm{d}\left(x_{4}, x_{7}, x_{8}\right)\right)
\end{gathered}
$$

occurring in $\left(b_{n}^{3 d}\left|W_{n}\right|^{2}\right)^{-1} \mu_{2,0}^{* * 0}$ can only be shown to be of asymptotic order $\mathcal{O}\left(b_{n}^{-d}\right)$. Assuming 
the fourth-order cumulant density $c^{(4)}$ to exist, the above integral turns into

$$
\begin{array}{r}
b_{n}^{d} \int_{\left(\mathbb{R}^{d}\right)^{8}} \frac{\left|W_{n} \cap\left(W_{n}-x_{5}\right)\right|\left|W_{n} \cap\left(W_{n}-x_{7}\right)\right|_{K}}{\left|W_{n}\right|^{2}} \mathbb{1}_{K}\left(t_{1}\right) \mathbb{1}_{K}\left(t_{2}\right) k\left(x_{2}\right) k\left(x_{4}\right) k\left(x_{6}\right) k\left(x_{8}\right) \\
\times c^{(4)}\left(b_{n} x_{2}+t_{1}, x_{5}, b_{n} x_{6}+x_{5}+t_{2}\right) c^{(4)}\left(b_{n} x_{4}+t_{1}, x_{7}, b_{n} x_{8}+x_{7}+t_{2}\right) \\
\mathrm{d} t_{1} \mathrm{~d} t_{2} \mathrm{~d} x_{2} \mathrm{~d} x_{4} \mathrm{~d} x_{5} \mathrm{~d} x_{6} \mathrm{~d} x_{7} \mathrm{~d} x_{8}
\end{array}
$$

by substitution. Due to $\sup _{u, v \in K \oplus b(o, \varepsilon)} \int_{\mathbb{R}^{d}}\left|c^{(4)}(u, w, v+w)\right| \mathrm{d} w<\infty$ for some $\varepsilon>0$ and since the kernel function is bounded with bounded support this term is of asymptotic order $\mathcal{O}\left(b_{n}^{d}\right)$. Likewise, the assumption $\sup _{u, v \in K \oplus b(o, \varepsilon)}\left|c^{(3)}(u, v)\right|<\infty$ is needed for showing that the integral

$$
\frac{1}{b_{n}^{3 d}\left|W_{n}\right|^{2}} \int_{\left(\mathbb{R}^{d}\right)^{6}} f_{1}\left(x_{1}, x_{2}, x_{3}, x_{4}\right) f_{1}\left(x_{1}, x_{5}, x_{3}, x_{6}\right) \gamma^{(3)}\left(\mathrm{d}\left(x_{1}, x_{2}, x_{5}\right)\right) \gamma^{(3)}\left(\mathrm{d}\left(x_{3}, x_{4}, x_{6}\right)\right)
$$

is of asymptotic order $\mathcal{O}\left(b_{n}^{d}\right)$ as $n \rightarrow \infty$.

The term $\mu_{1,1}^{* * 1}$ is of asymptotic order $\mathcal{O}\left(b_{n}^{2 d+1}\left|W_{n}\right|\right)$ and $\mu_{0,2}^{* * 2}$ is of asymptotic order $\mathcal{O}\left(b_{n}^{2 d+2}\left|W_{n}\right|\right)$ which can be shown by using the finiteness of the total variations $\left\|\gamma_{\text {red }}^{(k)}\right\|, k=2, \ldots, 6$, the upper bound (7) for the error term $\left|R_{n}\right|$ occurring in the function $\tilde{f}_{2}$, and the boundedness conditions on the kernel function. Together with the representation (10) this leads to the asymptotic representation

$$
\begin{aligned}
b_{n}^{d}\left|W_{n}\right|^{2} \Gamma_{2}\left(I_{n}(K)\right)= & 2 \lambda^{2} \int_{\mathbb{R}^{d}}(k * k)^{2}(t) \mathrm{d} t\left(\int_{K} \varrho^{2}(t) \mathrm{d} t+\int_{K \cap(-K)} \varrho^{2}(t) \mathrm{d} t\right) \\
& +\mathcal{O}\left(b_{n}^{d \wedge 2}\right)+\mathcal{O}\left(\left(b_{n}^{d}\left|W_{n}\right|\right)^{-1}\right)+\mathcal{O}\left(b_{n}^{d+4}\left|W_{n}\right|\right) .
\end{aligned}
$$

Now the assumption $b_{n}^{d+4}\left|W_{n}\right| \rightarrow 0$ implies the assertion.

Proof of Theorem 3.3. The asymptotic variance of $b_{n}^{d / 2}\left|W_{n}\right|\left(I_{n}(K)-\mathbb{E} I_{n}(K)\right)$ has already been determined in Lemma 3.2. We will prove asymptotic normality by showing that the $k$ th cumulant of $b_{n}^{d / 2}\left|W_{n}\right|\left(I_{n}(K)-\mathbb{E} I_{n}(K)\right)$ converges to zero for all $k \geq 3$.

In Lemma 5.2 we derived a representation of the $k$ th cumulant of $I_{n}(K)$ by indecomposable and irreducible integrals. Now we will show that the $k$ th cumulant of $b_{n}^{d / 2}\left|W_{n}\right|\left(I_{n}(K)-\mathbb{E} I_{n}(K)\right)$ is of order $O\left(\left(b_{n}^{d}\right)^{k / 2-1}+b_{n}^{4+\frac{k}{2} d}\left|W_{n}\right|\right)$ as $n \rightarrow \infty$ for $k \geq 2$. This implies the cumulants of order three and higher to converge to zero.

We will use the representation $\Gamma_{k}\left(I_{n}(K)\right)$ derived in Lemma 5.2 and determine the asymptotic order of the terms $\mu_{k-j, j}^{* * j}$ for $j=0, \ldots, k$. It is essential that the integrals in $\mu_{k-j, j}^{* * j}$ are neither decomposable nor reducible.

Consider an integral $I_{\mathcal{P}, \mathcal{Q}}($.$) in \mu_{k-j, j}^{* * j}, j=0, \ldots, k$, see (1). Let $V$ be the set of integration variables occurring in the integral and define the set of argument pairs

$$
\begin{aligned}
\mathcal{V}:=\{\{v, w\} \subseteq V: & \text { the integrand of } I_{\mathcal{P}, \mathcal{Q}}(.) \text { contains } \\
& \text { a term } \left.f_{1}(v, w, . . .), \text { a term } f_{1}(., ., v, w), \text { or a term } f_{2}(v, w)\right\} .
\end{aligned}
$$

Now we define a linkage relation on $\mathcal{V}$. Two argument pairs $\{v, w\},\{x, y\} \in \mathcal{V}$ are said to be linked (notation: $\{v, w\} \smile\{x, y\}$ ) if at least one of the following conditions is satisfied: 
(i) The argument pairs $\{v, w\},\{x, y\}$ have a common element, that is, $\{v, w\} \cap\{x, y\} \neq \emptyset$.

(ii) The integral $I_{\mathcal{P}, \mathcal{Q}}($.$) involves an integration \gamma^{(i)}\left(\mathrm{d}\left(v_{1}, \ldots, v_{i}\right)\right)$ for some $i \geq 2$ and some $v_{1}, \ldots, v_{i} \in V$ such that $\{v, w\} \cap\left\{v_{1}, \ldots, v_{i}\right\} \neq \emptyset$ and $\{x, y\} \cap\left\{v_{1}, \ldots, v_{i}\right\} \neq \emptyset$.

(iii) The integral $I_{\mathcal{P}, \mathcal{Q}}($.$) involves an integration \gamma^{(1)}\left(\mathrm{d} v_{0}\right) \gamma^{(i)}\left(\mathrm{d}\left(v_{1}, \ldots, v_{i}\right)\right)$ for some $i \geq 1$ and $v_{0}, \ldots, v_{i} \in V$ such that $\{v, w\} \cap\left\{v_{0}, \ldots, v_{i}\right\} \neq \emptyset$ and $\{x, y\} \cap\left\{v_{0}, \ldots, v_{i}\right\} \neq \emptyset$.

Note that the relation $\smile$ is reflexive and symmetric.

The maximal asymptotic order of each integration of linked argument pairs with $\ell$ arguments is $O\left(\left(b_{n}^{d}\right)^{\left\lceil\frac{\ell}{2}\right\rceil}\left|W_{n}\right|\right)$. After reduction of the factorial cumulant measures we make use of the existence of the cumulant densities. There are at least $\left\lceil\frac{\ell}{2}\right\rceil$ kernel functions $k$. By substitution of the arguments of the kernel functions $k$ we get a factor $b_{n}^{d}$ for each function. Furthermore there is exactly one variable occurring only in the indicator functions $\mathbb{1}_{W_{n}}$ (this is due to the integral's indecomposability and irreducibility). Integration over this variable yields the factor $\left|W_{n}\right|$. Because of the boundedness of the total variations the integrals over the cumulant densities are also bounded. Therefore we obtain the order $\mathcal{O}\left(\left(b_{n}^{d}\right)^{\left\lceil\frac{\ell}{2}\right\rceil}\left|W_{n}\right|\right)$ for each integration over $\ell$ linked argument pairs. Note that without the existence of the cumulant densities one can only derive the order $\mathcal{O}\left(\left|W_{n}\right|\right)$. For determining the order of the whole integral we also have to take into account that some of the arguments $t$ of the functions $\mathbb{1}_{K}(t)$ can be substituted, where each substitution produces a factor $b_{n}^{d}$. Thus the highest-order terms are those in which as many argument pairs as possible are not linked.

We will now use the concept of a cyclic linkage. Consider a product

$$
\prod_{i=1}^{m} f_{1}\left(p_{i}, q_{i}\right)
$$

occurring in the integrand of $I_{\mathcal{P}, \mathcal{Q}}($.$) and involving the argument pairs p_{1}, q_{1}, \ldots, p_{m}, q_{m} \in \mathcal{V}$. (Here $f_{1}(p, q)$ with argument pairs $p=\{u, v\}, q=\{x, y\}$ is understood as $f_{1}(u, v, x, y)$.) This product is said to be cyclically linked if there are an enumeration $r_{1}, \ldots, r_{2 m}$ of $\left\{p_{1}, q_{1}, \ldots, p_{m}, q_{m}\right\}$ and a permutation $\pi$ of $\{1, \ldots, m\}$ such that $\left\{r_{2 i-1}, r_{2 i}\right\}=\left\{p_{\pi(i)}, q_{\pi(i)}\right\}$ for all $i=1, \ldots, m$ and such that

$$
r_{2 i} \smile r_{2 i+1 \bmod 2 m} \text { for all } i \in\{1, \ldots, m\}
$$

is an exhaustive list of the links between the argument pairs $p_{1}, q_{1}, \ldots, p_{m}, q_{m}$.

We will now investigate the highest-order terms in $\mu_{k-j, j}^{* * j}$ for $j=0, \ldots, k$.

Let $j=0$. Then the integrands of all highest-order integrals in $\mu_{k, 0}^{* * 0}$ are cyclically linked. As an example consider the integral

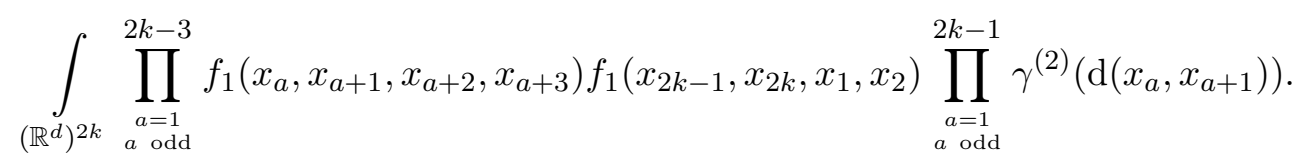

By disintegration and substitution we get 


$$
\begin{aligned}
\lambda^{k}\left|W_{n}\right|^{k} \int_{\left(\mathbb{R}^{d}\right)^{k}} \prod_{\substack{a=1 \\
a \text { odd }}}^{2 k-3} k\left(\frac{x_{a+1}-t_{a}}{b_{n}}\right) k\left(\frac{x_{a+3}-t_{a}}{b_{n}}\right) \mathrm{d} t_{a} \\
\times \int_{K} k\left(\frac{x_{2 k}-t_{2 k-1}}{b_{n}}\right) k\left(\frac{x_{2}-t_{2 k-1}}{b_{n}}\right) \mathrm{d} t_{2 k-1} \prod_{\substack{a=1 \\
a \text { odd }}}^{2 k-1} \gamma_{\text {red }}^{(2)}\left(\mathrm{d} x_{a+1}\right) \\
=\left(b_{n}^{d}\right)^{k}\left|W_{n}\right|^{k} \lambda^{k} \int_{\left(\mathbb{R}^{d}\right)^{2 k}} \prod_{\substack{a=1 \\
a \text { odd }}}^{2 k-3} \mathbb{1}_{K}\left(t_{a}\right) k\left(x_{a+1}\right) k\left(x_{a+3}+\frac{t_{a+2}-t_{a}}{b_{n}}\right) \mathbb{1}_{K}\left(t_{2 k-1}\right) k\left(x_{2 k}\right) \\
\times k\left(x_{2}+\frac{t_{1}-t_{2 k-1}}{b_{n}}\right) \prod_{\substack{a=1 \\
a \text { odd }}}^{2 k-1} c^{(2)}\left(b_{n} x_{a+1}+t_{a}\right) \\
\mathrm{d} x_{2} \mathrm{~d} x_{4} \ldots \mathrm{d} x_{2 k} \mathrm{~d} t_{1} \mathrm{~d} t_{3} \ldots \mathrm{d} t_{2 k-1} .
\end{aligned}
$$

By substituting $\tilde{t}_{a+2}=\frac{t_{a+2}-t_{a}}{b_{n}}, a=3,5, \ldots, 2 k-1$, we see that this is equal to

$$
\begin{array}{r}
\left(b_{n}^{d}\right)^{2 k-1}\left|W_{n}\right|^{k} \lambda^{k} \int_{\left(\mathbb{R}^{d}\right)^{2 k}} \prod_{\substack{a=1 \\
a \text { odd }}}^{2 k-3} \mathbb{1}_{K}\left(b_{n} t_{a+2}+t_{1}\right) k\left(x_{a+1}\right) k\left(x_{a+3}+t_{a+2}-t_{a}\right) \mathbb{1}_{K}\left(t_{1}\right) k\left(x_{2 k}\right) \\
\quad \times k\left(x_{2}-t_{2 k-1}\right) \prod_{\substack{a=3 \\
a \text { odd }}}^{2 k-1} c^{(2)}\left(b_{n} x_{a+1}+b_{n} t_{a}+t_{1}\right) c^{(2)}\left(b_{n} x_{2}+t_{1}\right) \\
\quad \mathrm{d} x_{2} \mathrm{~d} x_{4} \ldots \mathrm{d} x_{2 k} \mathrm{~d} t_{1} \mathrm{~d} t_{3} \ldots \mathrm{d} t_{2 k-1} .
\end{array}
$$

The second-order cumulant density $c^{(2)}$ is continuous since the product density is continuous in $K \oplus b(o, \varepsilon)$ for some $\varepsilon>0$. Hence the above-mentioned integral is of order $O\left(\left(b_{n}^{d}\right)^{2 k-1}\left|W_{n}\right|^{k}\right)$. Analogous arguments apply to the other terms in $\mu_{k, 0}^{* *}$.

Now let $j=1$. Then each integrand of a highest-order term in $\mu_{k-1,1}^{* * 1}$ is a product of two parts: First, a cyclically linked product of $k-1$ instances of $f_{1}$, and second, one instance of the function $\tilde{f}_{2}$ whose argument pair is linked to at least one argument pair from the first part. One of these highest-order integrals is

$$
\int_{\left(\mathbb{R}^{d}\right)^{2 k-2}} \prod_{\substack{a=1 \\ a \text { odd }}}^{2 k-5} f_{1}\left(x_{a}, x_{a+1}, x_{a+2}, x_{a+3}\right) f_{1}\left(x_{2 k-3}, x_{2 k-2}, x_{1}, x_{2}\right) \tilde{f}_{2}\left(x_{1}, x_{2}\right) \prod_{\substack{a=1 \\ a \text { odd }}}^{2 k-3} \alpha^{(2)}\left(\mathrm{d}\left(x_{a}, x_{a+1}\right)\right) .
$$

By applying disintegration and substitution as above and taking advantage of the upper bound (7) for $\left|R_{n}\right|$ due to the uniform Lipschitz-continuity of the first-order partial derivatives of $\varrho$ in $K \oplus b(o, \varepsilon)$ for some $\varepsilon>0$, one finds the above-mentioned integral to be of asymptotic order $O\left(b_{n}\left(b_{n}^{d}\right)^{2 k-2}\left|W_{n}\right|^{k-1}\right)$. Analogous arguments apply to the remaining integrals.

Next let $j=2$. Then each integrand of a highest-order term in $\mu_{k-2,2}^{* * 2}$ is a product of two parts: First, a cyclically linked product of $k-2$ instances of $f_{1}$, and second, a product of two instances of the function $\tilde{f}_{2}$ whose argument pairs are both linked to argument pairs from the first part. For example, the integral

$$
\int_{\left(\mathbb{R}^{d}\right)^{2 k-4}} \prod_{\substack{a=1 \\ a \text { odd }}}^{2 k-7} f_{1}\left(x_{a}, x_{a+1}, x_{a+2}, x_{a+3}\right) \tilde{f}_{2}\left(x_{2 k-5}, x_{2 k-4}\right) \tilde{f}_{2}\left(x_{1}, x_{2}\right) \prod_{\substack{a=1 \\ a \text { odd }}}^{2 k-5} \alpha^{(2)}\left(\mathrm{d}\left(x_{a}, x_{a+1}\right)\right)
$$


is of asymptotic order $\mathcal{O}\left(b_{n}^{2}\left(b_{n}^{d}\right)^{2 k-2}\left|W_{n}\right|^{k-1}\right)$ and hence one of the highest-order terms for the case $j=2$.

For $j=3, \ldots, k-1$ one obtains the asymptotic order $\mathcal{O}\left(b_{n}^{j}\left(b_{n}^{d}\right)^{2 k-j}\left|W_{n}\right|^{k-j+1}\right)$ by analogous considerations.

Finally, in the case $j=k$ all integrands of the integrals in $\mu_{0, k}^{* * k}$ are products of $k$ instances of the function $\tilde{f}_{2}$. Since these integrals are indecomposable the argument pairs occurring in the integrand can be enumerated as $p_{1}, \ldots, p_{k}$ such that $p_{i} \smile p_{i+1}$ for $i=1, \ldots, k-1$. Hence the term $\mu_{0, k}^{* * k}$ is of order $\mathcal{O}\left(b_{n}^{k}\left(b_{n}^{d}\right)^{k}\left|W_{n}\right|\right)$.

Altogether we have

$$
\begin{gathered}
\mu_{k, 0}^{* * 0}=\mathcal{O}\left(\left(b_{n}^{d}\right)^{2 k-1}\left|W_{n}\right|^{k}\right), \\
\mu_{k-1,1}^{* * 1}=\mathcal{O}\left(b_{n}\left(b_{n}^{d}\right)^{2 k-2}\left|W_{n}\right|^{k-1}\right),
\end{gathered}
$$

and

$$
\mu_{k-j, j}^{* * j}=\mathcal{O}\left(b_{n}^{j}\left(b_{n}^{d}\right)^{2 k-j}\left|W_{n}\right|^{k-j+1}\right) \quad \text { for } j=2, \ldots, k .
$$

Together with Lemma 5.2 the $k$ th cumulant hence satisfies

$$
\Gamma_{k}\left(I_{n}(K)\right)=\mathcal{O}\left(b_{n}^{-d}\left|W_{n}\right|^{-k}\right)+2 k \mathcal{O}\left(b_{n}^{2-d}\left|W_{n}\right|^{-k}\right)+\sum_{j=2}^{k}\left(\begin{array}{c}
k \\
j
\end{array}\right) 2^{j} \mathcal{O}\left(b_{n}^{2 j}\left|W_{n}\right|^{1-k}\right) .
$$

As a result the $k$ th cumulant of $b_{n}^{d / 2}\left|W_{n}\right|\left(I_{n}(K)-\mathbb{E} I_{n}(K)\right)$ is of order $O\left(\left(b_{n}^{d}\right)^{k / 2-1}+b_{n}^{4+\frac{k}{2} d}\left|W_{n}\right|\right)$ for $k \geq 2$. Due to the assumption $b_{n}^{d+4}\left|W_{n}\right| \underset{n \rightarrow \infty}{\longrightarrow} 0$ the $k$ th cumulant of $b_{n}^{d / 2}\left|W_{n}\right|\left(I_{n}(K)-\mathbb{E} I_{n}(K)\right)$ converges to zero for every $k \geq 3$. This proves normal convergence.

\section{References}

[1] Baddeley, A., Turner, R., Møller, J., and Hazelton, M. (2005). Residual analysis for spatial point processes (with discussion). Journal of the Royal Statistical Society, series B, 67(5):617666.

[2] Brillinger, D.R. (1975). Statistical inference for stationary point processes. In Puri, M. L., editor, Stochastic Processes and Related Topics, volume 1 of Proceedings of the Summer Research Institute on Statistical Inference for Stochastic Processes, pages 55-99. Academic Press, New York.

[3] Cressie, N.A.C. (1993). Statistics for Spatial Data. Wiley \& Sons, New York.

[4] Daley, D.J. and Vere-Jones, D. (2008). An Introduction to the Theory of Point Processes. Vol. II: Genereral Theory and Structure. Springer, New York.

[5] Diggle, P.J. (1979). On parameter estimation and goodness-of-fit testing for spatial point patterns. Biometrics, 35(1):87-101.

[6] Diggle, P.J. (2003). Statistical Analysis of Spatial Point Patterns. 2nd ed. Arnold, London.

[7] Grabarnik, P. and Chiu, S.N. (2002). Goodness-of-fit test for complete spatial randomness against mixtures of regular and clustered spatial point processes. Biometrika, 89(2):411-421. 
[8] Guan, Y. (2008). A KPSS test for stationarity for spatial point processes. Biometrics, 64(3):800-806.

[9] Hall, P. (1984). Central limit theorem for integrated square error of multivariate nonparametric density estimators. Journal of Multivariate Analalysis, 14:1-16.

[10] Heinrich, L. (1988). Asymptotic Gaussianity of some estimators for reduced factorial moment measures and product densities of stationary poisson cluster processes. Statistics, 19:87-106.

[11] Heinrich, L. and Schmidt, V. (1985). Normal convergence of multidimensional shot noise and rates of this convergence. Advances in Applied Probability, 17:709-730.

[12] Ho, L.P. and Chiu, S.N. (2006). Testing the complete spatial randomness by Diggle's test without an arbitrary upper limit. Journal of Statistical Computation and Simulation, $76(7): 585-591$.

[13] Illian, J., Penttinen, A., Stoyan, H., and Stoyan, D. (2008). Statistical Analysis and Modelling of Spatial Point Patterns. Wiley \& Sons, Chichester.

[14] Ivanoff, G. (1982). Central limit theorem for point processes. Stochastic Processes and their Applications, 12:171-186.

[15] Jolivet, E. (1981). Central limit theorem and convergence of empirical processes for stationary point processes. In Point processes and Queueing Problems. P. Bartfai and J. Tomko, NorthHolland, Amsterdam, 117 - 161.

[16] Jolivet, E. (1984). Upper bounds of the speed of convergence of moment density estimators for stationary point processes. Metrika, 31:349-360.

[17] Krickeberg, K. (1982). Processus ponctuels en statistique. In École d'Éte de Probabilités de Saint-Flour X - 1980, volume 929 of Lecture Notes in Mathematics, pages 205-313. Springer, Berlin.

[18] Leonov, V.P. and Shiryaev, A.N. (1959). On a method of calculation of semi-invariants. Theory of Probability and its Applications, 4(3):319-329.

[19] Stoyan, D., Kendall, W.S., and Mecke, J. (1995). Stochastic Geometry and Its Applications. 2nd ed. Wiley \& Sons, New York.

[20] Stoyan, D. and Stoyan, H. (2000). Improving ratio estimators of second order point process characteristics. Scandinavian Journal of Statistics, 27:641-656.

[21] Takahata, H. and Yoshihara, K. (1987). Central limit theorems for integrated square error of nonparametric density estimators based on absolutely regular random sequences. Yokohama Mathematical Journal, 35:95-111.

[22] Zimmerman, D.L. (1993). A bivariate Cramér-von Mises type of test for spatial randomness. Applied Statistics, 42(1):43-54. 